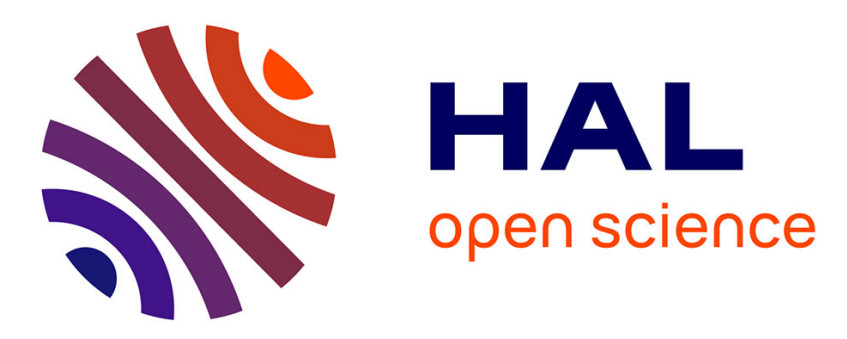

\title{
Non invasive blood flow features estimation in cerebral arteries from uncertain medical data
}

\author{
Rajnesh Lal, Franck Nicoud, Emmanuelle Le Bars, Jeremy Deverdun, \\ François Molino, Vincent Costalat, Bijan Mohammadi
}

\section{- To cite this version:}

Rajnesh Lal, Franck Nicoud, Emmanuelle Le Bars, Jeremy Deverdun, François Molino, et al.. Non invasive blood flow features estimation in cerebral arteries from uncertain medical data. Annals of Biomedical Engineering, 2017, 45 (11), pp.2574-2591. 10.1007/s10439-017-1904-7 . hal-01575417

\section{HAL Id: hal-01575417 \\ https://hal.science/hal-01575417}

Submitted on 19 Aug 2017

HAL is a multi-disciplinary open access archive for the deposit and dissemination of scientific research documents, whether they are published or not. The documents may come from teaching and research institutions in France or abroad, or from public or private research centers.
L'archive ouverte pluridisciplinaire HAL, est destinée au dépôt et à la diffusion de documents scientifiques de niveau recherche, publiés ou non, émanant des établissements d'enseignement et de recherche français ou étrangers, des laboratoires publics ou privés.

$$
\text { Copyright }
$$




\title{
Non invasive blood flow features estimation in cerebral arteries from uncertain medical data
}

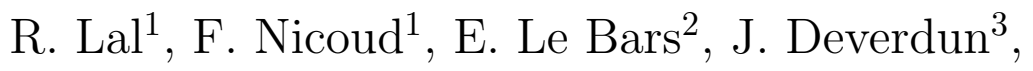 \\ F. Molino ${ }^{4}$, V. Costalat ${ }^{2}$ and B. Mohammadi ${ }^{1}$
}

affiliations: ${ }^{1}$ Institut Montpelliérain Alexander Grothendieck (IMAG), Université de Montpellier, Montpellier, France.

${ }^{2}$ Institut d'Imagerie Fonctionnelle Humaine (I2FH) Hôpital Gui de Chauliac, Montpellier, France. Service de Neuroradiologie - Pôle tête et cou - Hôpital Gui de Chauliac, Montpellier, France.

${ }^{3}$ Unité I2FH, Institut d'Imagerie Fonctionnelle Humaine, Montpellier University Hospital Center, Gui de Chauliac Hospital, Montpellier, France.

${ }^{4}$ Laboratoire Charles Coulomb, Université de Montpellier, Montpellier, France.

abbreviated title: Non invasive blood pressure estimation from MRA\&MRI data

correspondence: Rajnesh Lal, IMAG, Université de Montpellier, CC051, 34095 Montpellier, France. e-mail: rajnesh.lal@etu.umontpellier.fr

\begin{abstract}
A methodology for non-invasive estimation of the pressure in internal carotid arteries is proposed. It uses data assimilation and Ensemble Kalman filters in order to identify unknown parameters in a mathematical description of the cerebral network. The approach uses patient specific blood flow rates extracted from Magnetic Resonance Angiography and Magnetic Resonance Imaging. This construction is necessary as the simulation of blood flows in complex arterial networks, such as the circle of Willis, is not straightforward because hemodynamic parameters are unknown as well as the boundary conditions necessary to close this complex system with many outlets. For instance, in clinical cases, the values of Windkessel model parameters or the Young's modulus and the thickness of the arteries are not available on per-patient cases. To make the approach computational efficient, a reduced order zero-dimensional compartment model is used for blood flow dynamics. Using this simplified model, the proof-of-concept study demonstrates how to use the EnKF as an optimization tool to find parameters and how to make the inverse hemodynamic problem tractable. The predicted blood flow rates in the internal carotid arteries and the predicted systolic and diastolic brachial blood pressures are found to be in good agreement with the clinical measurements.
\end{abstract}

Keywords: MRA, MRI, EnKF, reduced order compartment blood model, parameter estimation, circle of Willis, hemodynamic inverse problems 


\section{Introduction}

One of the key factors identified to be associated with the formation and the risk of rupture of a cerebral aneurysm is the blood pressure fluctuations in cerebral arteries ${ }^{1 / 3}$. The circle of Willis $(\mathrm{CoW})$ is a common place for aneurysms ${ }^{4}$. There have been many researches done on hemodynamics and the blood flow in the CoW ${ }^{5-7}$ focusing on the understanding of factors increasing the risk of stroke and the blood flow distribution in the brain. While methods and procedures are available for assessing and measuring the pressure in cerebral arteries, they are mostly based on invasive methods such as using a pressure-sensing catheter. Hasan et al. ${ }^{8}$ used dual-sensor microwires (ComboWire; Volcano Corporation) to measure systolic, diastolic, and mean pressure inside a cerebral aneurysm. A linear relationship between changes in radial and aneurysmal pressures was found. ComboWire was also used by Ferns et al. ${ }^{9}$ to get pressure measurements in the internal carotid artery of patients with an unruptured intracranial aneurysm. Because non-invasive pressure measurements in cerebral arteries are not available, the systolic-diastolic pressure variation in an aneurysm cannot be included in the risk of rupture analysis, although this quantity is obviously very relevant from a biomechanical point of view. Relying on a physical/numerical description of the cerebral hemodynamics to assess blood pressure non-invasively is thus an appealing alternative.

The analysis of the cardiovascular system can be carried out using one-dimensional $(1 \mathrm{D})^{10} \frac{12}{12}$ or multi-dimensional modelling $(2 \mathrm{D} \text { or } 3 \mathrm{D})^{13}$ or in a simplified manner using a lumped model (0D compartment model) 14 . It is possible to numerically solve the pressure and the blood flow rate wave system, taking place in a network of interconnected arteries representing all or part of the cardiovascular system. Lumped parameter models have been extensively developed and used over the years to study the blood circulation dynamics and for the assessment of hemodynamics (Ursino and Giannessi ${ }^{14}$, Milišic and Quarteroni ${ }^{[15}$, Pant et al. ${ }^{16}$, Ellwein et al. ${ }^{17}$, Pant et al. 18, Quarteroni et al. 19, Westerhof et al.20, Blanco et al. ${ }^{21}$ ) or to investigate cerebral hemodynamics (Liang et al. ${ }^{22}$, Gao et al. ${ }^{23}$, Olufsen et al. ${ }^{24}$, Pope et al. $\left.{ }^{251}\right)$.

Using imaging data such as Magnetic Resonance Angiography and Magnetic Resonance Imaging (MRA\&MRI), the geometric properties of each blood vessels such as diameter and length can be acquired. However, the structural properties of the vessels such as the wall thickness and the Young's modulus are difficult to identify. Still in patient-specific simulations, such unknown properties need to be estimated as well as the distal boundary conditions. These unknown parameters can be estimated using an algorithm based on a data assimilation technique such as an Unscented Kalman filter (UKF) ${ }^{26 \mid 27}$ or its variant reduced order unscented Kalman filter $(\mathrm{ROUKF})^{28129}$ or an Ensemble Kalman filter $(\mathrm{EnKF})^{30131}$. The idea of using the Kalman filter tool in data assimilation techniques is to improve the simulated results of a model through the estimation of the parameter values used in the numerical model. A general data assimilation technique uses available clinical observations (possibly corrupted by noise) on a given system such as brachial blood pressure, carotid flow rate, temporal pressure, aortic flow rate or cross-sectional area. A predictor-corrector method is applied to the set of uncertain parameters whose values are corrected taking into account the difference between the clinical observations and the measurements that are simulated using the blood flow model. The Kalman gain matrices 
are defined in Kalman filter tool to minimize the distance between clinical observations and measurements taking into account both the uncertainties in the model and in the measurements. Once the assimilation process is completed and the parameters are estimated, the interconnected network of arteries becomes a good approximation of patient specific cardiovascular system on which the morphological data are collected and hemodynamic observations are made. It is then possible to use the blood flow model to estimate the hemodynamic variables whose measurement is only possible invasively (e.g. to access aneurysm pressure). The outcome of data assimilation methods is (based on the patient-specific measurements) the development of numerical models by estimating hemodynamic parameters. In the process, model simulation is adapted to the patient-specific data resulting in reliably simulated measurements (predictions).

Recent works on inverse problems in hemodynamics using a 1D or multi-dimensional blood flow model include the work of Chabiniok et al. ${ }^{26}$, Lombardi ${ }^{28}$, Moireau et al. ${ }^{29}$, Lal

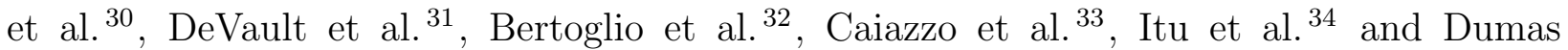
et al. .35 . In Chabiniok et al. $\stackrel{26}{ }$, Lombardi 28 , Moireau et al. 29 , Bertoglio et al. ${ }^{32}$ and Caiazzo et al. ${ }^{33}$ a ROUKF is used as a tool for data assimilation to estimate hemodynamic parameters such as elastic properties of arteries, arterial compliance and boundary condition parameters (Windkessel boundary parameters and reflection coefficients). In Lal

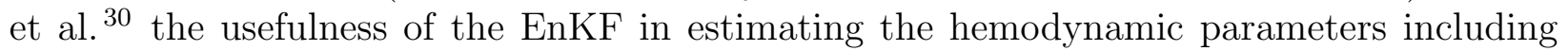
the arterial stiffness and boundary condition parameter (reflection coefficient) is demonstrated. Using in silico experiments, Lal et al. $\stackrel{30}{ }$ have extensively assessed the EnKF-based parameter estimation algorithm. In their approach, they have validated predicted pressure levels on physical phantoms. DeVault et al. ${ }^{31}$ have demonstrated the use of an EnKF to calibrate the boundary condition parameters of a network with 16 arteries in the CoW using a one-dimensional numerical model for the prediction of cerebral

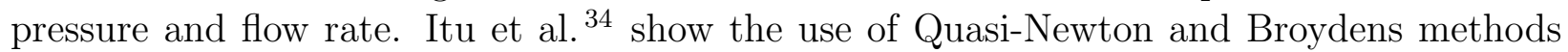
for the estimation of Windkessel boundary parameters. Dumas et al. $\stackrel{35}{ }$ have proposed a numerical approach to determine the distribution of the arterial stiffness in a subject-specific network of arteries. In the proposed approach, the optimal parameters of the one-dimensional model have been calibrated by solving an inverse problem.

Performing data assimilation with a compartment model (0D) has many advantages: the parameter estimation procedure is fast as the 0D model only takes a few seconds (depending on the arterial network) to run, the inverse problem can be run from several initial guesses of hemodynamic parameters without significant increase in computing cost and time and the sensitivity analysis is cheap. In Pant et al. $\frac{[16}{16}$ an estimation of lumped model parameters (Windkessel) from uncertain clinical data using the unscented Kalman filter is proposed. The method is extended to a patient specific parameter estimation of a lumped parameter model of blood circulation for single-ventricle shunt physiology in Pant et al. 27. In Ellwein et al. 17 a compartment model is used to predict arterial and venous blood pressures including the volume of the heart using 11 compartments representing the systemic circulation. The model parameters are estimated using a non-linear optimization technique. In Pope et al. ${ }^{25}$ estimation and identification of parameters in a lumped cerebrovascular model are presented. The parameters are estimated using Gauss Newton gradient-based nonlinear optimization technique.

This work proposes to estimate the pressure variations for an arterial network 
representative of the cerebral circulation using an integrated observation/simulation/assimilation procedure to exploit available MRA\&MRI observation data by EnKF parameter estimation techniques using reduced order blood flow models. In the current work, an EnKF-based parameter estimation algorithm is coupled to a 0D compartment network in order to 1- estimate the model parameters (arterial stiffness and distal boundary conditions) of a patient (healthy volunteer) specific complex network including the CoW and 2- assess the blood pressure fluctuations in the whole network once the parameters have been adapted. Technically demanding and not free of risk, invasive pressure measurements can hardly be included in proof-of-concept studies for validation purposes. The accuracy of the predictions is thus assessed by comparing the model predicted and clinically measured systolic and diastolic pressures (using a cuff-based oscillometry) at the brachial artery. To the authors knowledge, this configuration to estimate hemodynamic parameters and predict blood pressure in cerebral arteries is relatively new with respect to the application of data assimilation to a complex arterial network including the CoW, the number of arteries (33) in the network, and the coupling of EnKF to the 0D model.

The outline of the paper is as follows: First, a review of the 0D lumped compartment model which will be used to represent the cardiovascular network is presented. Next, the ensemble Kalman data assimilation technique is reviewed and the algorithm for parameter estimation given. Then, the application of the procedure to the test cases with synthetic and actual clinical data is shown. The clinical data correspond to the cerebral network, including the complete circle of Willis (CoW), of a healthy subject.

\section{Materials and Methods}

\section{$2.1 \quad$ OD Model for Cardiovascular system}

The human cardiovascular system can be modelled using an electrical analogy to represent different mechanical properties of arteries ${ }^{14}$ 16/19. In this model (called compartment models, 0D models or lumped parameter models), the arterial network is divided into different compartments comprising a resistor (resistance of blood due to blood viscosity, $R$ ), an inductor (blood inertance $L$ ) and a capacitor (compliance of the artery, $C$ ).

The four common compartmental configurations in 0D models are $\mathscr{L}$ network element, inverted $\mathscr{L}$ network element, $\mathcal{T}$ element and $\pi$ element networks. The use of the four configurations depends on the prescribed boundary conditions and the detailed analysis of the configurations can be found in Milišić and Quarteroni ${ }^{15}$ and Quarteroni et al.19. In the current work, an inverted $\mathscr{L}$ network element (Figure 1) is used. In this element, the upstream pressure $P_{\text {in }}$ and the downstream flow rate $q_{\text {out }}$ are used as the boundary conditions.

The spatial variation of parameters $(R, L$, and $C)$ in lumped parameter models is neglected in each spatial compartment and thus the parameters are assumed to be uniform. For a single compartment assuming an incompressible Newtonian fluid, the governing equations for the 0D model (inverted $\mathscr{L}$ network element) relating the variables $R, L$ and 


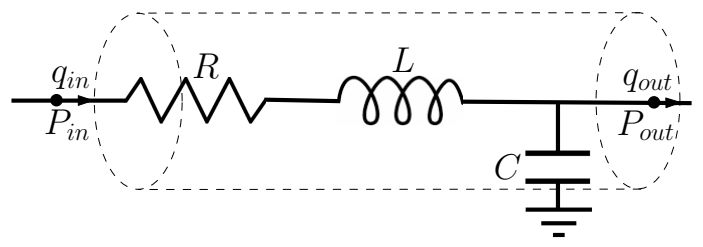

Figure 1: Single compartment circuit representation

$C$ and representing mass and momentum conservation read: $\underline{14|15| 19}$

$$
\begin{aligned}
C \frac{d P_{\text {out }}}{d t} & =q_{\text {in }}-q_{\text {out }} \\
L \frac{d q_{\text {in }}}{d t}+R q_{\text {in }} & =P_{\text {in }}-P_{\text {out }}
\end{aligned}
$$

where $P_{\text {in }}, q_{\text {in }}$ and $P_{\text {out }}, q_{\text {out }}$ are the blood pressure and flow rate at the inlet and outlet of the compartment (artery) respectively. The inertance $L$ expresses the inertia within the vessel and if its effect is ignored, the flow rate is then given by $q_{\text {in }}=\left(P_{\text {in }}-P_{\text {out }}\right) / R$.

The parameters $R, L$ and $C$ for each of the compartment representing different arterial segments are calculated using the following equations ${ }^{16}$ : Hagen-Poiseuille law for resistance, $R=8 \mu l / \pi r^{4}, L=\rho l / \pi r^{2}$ and $C=3 \pi r^{3} l / 2 E h$, where $E, h, \rho, \mu, l$ and $r$ is the Young's modulus, arterial wall thickness, the blood density, the blood viscosity, the length of the arterial segment and, the radius of the artery respectively.

Each segment of the arterial network including the CoW is represented with a reduced order 0D model consisting of the three elements $R, L$, and $C$ (see Figure 2 where each of the arterial segment is represented by a single compartment). In this way, a distributed lumped parameter model is developed for the full network in which multiple lumped compartments are connected in series.

At the bifurcation, boundary conditions are prescribed by enforcing conservation of mass and continuity of pressure. At the outlet of each terminal compartment, the blood flow model is coupled to the three-element Windkessel model (WK3-lumped parameter model) 7111 to include the effect of the downstream vasculature. In the WK3 model, the instantaneous blood pressure and the flow rate are related as follows:

$$
\frac{d p(t)}{d t}+\frac{p}{R_{D} C}=R_{P} \frac{d q(t)}{d t}+\frac{q\left(R_{P}+R_{D}\right)}{R_{D} C}
$$

where $p$ is the instantaneous pressure at the inlet of the WK3 model, $q$ is the instantaneous flow rate, $R_{P}$, and $R_{D}$ are the proximal (characteristic) and distal resistance respectively of the vascular beds, and $C$ is the compliance. $R_{T}=R_{P}+R_{D}$ represents the total resistance of a peripheral bed.

Of course, more sophisticated physical models can be considered. But, this is not central to our discussion. What we want to show in this paper is that this simple model permits to give reasonable results after inversion. 


\subsection{Estimation of hemodynamic parameters using an Ensemble Kalman Filter}

The literature on ensemble Kalman filter algorithms is vast ${ }^{36137}$. The reader is referred to Lal et al. ${ }^{30}$ for a detailed mathematical analysis of the EnKF implementation. Below the algorithm used to estimate the hemodynamic model parameters is summarised.

The EnKF is a suboptimal estimator for problems that involves nonlinear models of higher order, where an ensemble of states is used to predict the error statistics ${ }^{30}$. The unknown hemodynamic parameters $\mathbf{x} \in \mathbb{R}^{n}$, are treated as special state variables whose evolutions are defined using a random walk model, $\mathbf{x}_{k+1}=\mathbf{x}_{k}+\tau_{k}$, where $\mathbf{x}_{k}$ denotes the state variable at time step $t_{k} . \tau_{k} \sim \mathcal{N}\left(0, \mathbf{T}_{k}\right)$ is a small Gaussian random perturbation with a variance $\mathbf{T}$. The initial forecast ensemble of parameters $\mathbf{x}_{k}^{f_{i}}$ for $i=1, \ldots, q_{\text {ens }}$ is assumed to be available at time $t_{k} . f_{i}$ denotes the initial $i^{\text {th }}$ forecast member of an ensemble of size $q_{\text {ens }}$ which is used to determine the forecast error covariance matrix in EnKF. The parameter estimation algorithm uses a set of nobs observations such as blood pressure, blood flow rates, flow velocity or arterial wall movements to improve a set of given initial estimate of the hemodynamic parameters, $\mathbf{x}$. A set of output vector, $\mathbf{Y}_{k}=\left(\mathbf{y}_{k}^{f_{1}}, \ldots, \mathbf{y}_{k}^{f_{\text {ens }}}\right) \in \mathbb{R}^{q_{\text {ens }} \times n o b s}$, is generated at time $t_{k}$. Each member, $\mathbf{y}_{k}^{f_{i}}$, is defined by $\mathbf{y}_{k}^{f_{i}}=\mathcal{H}\left(\mathbf{x}^{f i}\right)$, where $\mathcal{H}$ is the nonlinear measurement function defined by the blood flow model describing the relation between measurements and parameters. An ensemble of perturbed observations, $\mathbf{y}_{k}^{i}$ (for $\left.i=1, \ldots, q_{\text {ens }}\right)$ is generated by adding perturbations to the original observation vector $\mathbf{y}_{k} \in$ $\mathbb{R}^{\text {nobs }}$ :

$$
\mathbf{y}_{k}^{i}=\mathbf{y}_{k}+\mathbf{e}_{k}^{i}, \quad i=1, \ldots, q_{\mathrm{ens}},
$$

where $\mathbf{e}_{k}^{i} \in \mathbb{R}^{\text {nobs }}$ is a random vector drawn from the zero mean Gaussian distribution with a specified variance. The discrepancies between perturbed observations and measurements are then used to update parameters using:

$$
\mathbf{x}_{k}^{\mathrm{a}_{i}}=\mathbf{x}_{k}^{\mathrm{f}_{i}}+\mathbf{K}_{k}\left[\mathbf{y}_{k}^{i}-\mathbf{y}_{k}^{\mathrm{f}_{i}}\right], i=1, \ldots, q_{\mathrm{ens}},
$$

where $\mathrm{a}_{i}$ represents the updated (assimilated) parameters and $\mathbf{K}_{k}$ is the Kalman gain matrix. The matrix $\mathbf{K}_{k}$ is defined by three error covariance matrices:

$$
\mathbf{K}_{k}=\mathbf{P}_{\mathbf{x y}_{k}}^{\mathrm{f}}\left(\mathbf{P}_{\mathbf{y y}_{k}}^{\mathrm{f}}+\mathbf{R}_{k}\right)^{-1} .
$$

$\mathbf{R}_{k}$ is the diagonal measurement error covariance matrix defined by

$$
\mathbf{R}_{\mathbf{k}}=\operatorname{diag}\left[\frac{1}{q_{\mathrm{ens}}-1} \mathbf{E E}^{\mathrm{T}}\right], \quad \mathbf{E}=\left[\mathbf{e}_{k}^{1}, \ldots, \mathbf{e}_{k}^{q_{\mathrm{ens}}}\right] .
$$

The other two error covariance matrices are given by ${ }^{30}$

$$
\mathbf{P}_{\mathbf{x y}_{k}}^{\mathrm{f}}=\frac{1}{q_{\text {ens }}-1} \sum_{i=1}^{q_{\text {ens }}}\left[\mathbf{x}_{k}^{\mathrm{f}_{i}}-\overline{\mathbf{x}}_{k}^{\mathrm{f}}\right]\left[\mathbf{y}_{k}^{\mathrm{f}_{i}}-\overline{\mathbf{y}}_{k}^{\mathrm{f}}\right]^{\mathrm{T}} ; \mathbf{P}_{\mathbf{y} \mathbf{y}_{k}}^{\mathrm{f}}=\frac{1}{q_{\text {ens }}-1} \sum_{i=1}^{q_{\text {ens }}}\left[\mathbf{y}_{k}^{\mathrm{f}_{i}}-\overline{\mathbf{y}}_{k}^{\mathrm{f}}\right]\left[\mathbf{y}_{k}^{\mathrm{f}_{i}}-\overline{\mathbf{y}}_{k}^{\mathrm{f}}\right]^{\mathrm{T}}
$$

where $\overline{\mathbf{x}}_{k}^{\mathrm{f}}=\frac{1}{q_{\text {ens }}} \sum_{i=1}^{q_{\text {ens }}} \mathbf{x}_{k}^{\mathrm{f}_{i}}$ and $\overline{\mathbf{y}}_{k}^{\mathrm{f}}=\frac{1}{q_{\text {ens }}} \sum_{i=1}^{q_{\text {ens }}} \mathcal{H}\left(\mathbf{x}_{k}^{\mathrm{f}_{i}}\right)$. The ensemble updated parameters (4) at time $t=k$ is then cycled in time and the parameter estimation using the EnKF can 
be stopped upon reaching some finite convergence criterion. At convergence, the mean of the ensemble is taken as the best estimate of the parameters. The parameter estimation algorithm is summarised in Algorithm $1_{1}^{130}$. Henceforth, nobs will refer to the number of locations on an arterial network where a time series of observations such as pressure values or blood flow rate is available.

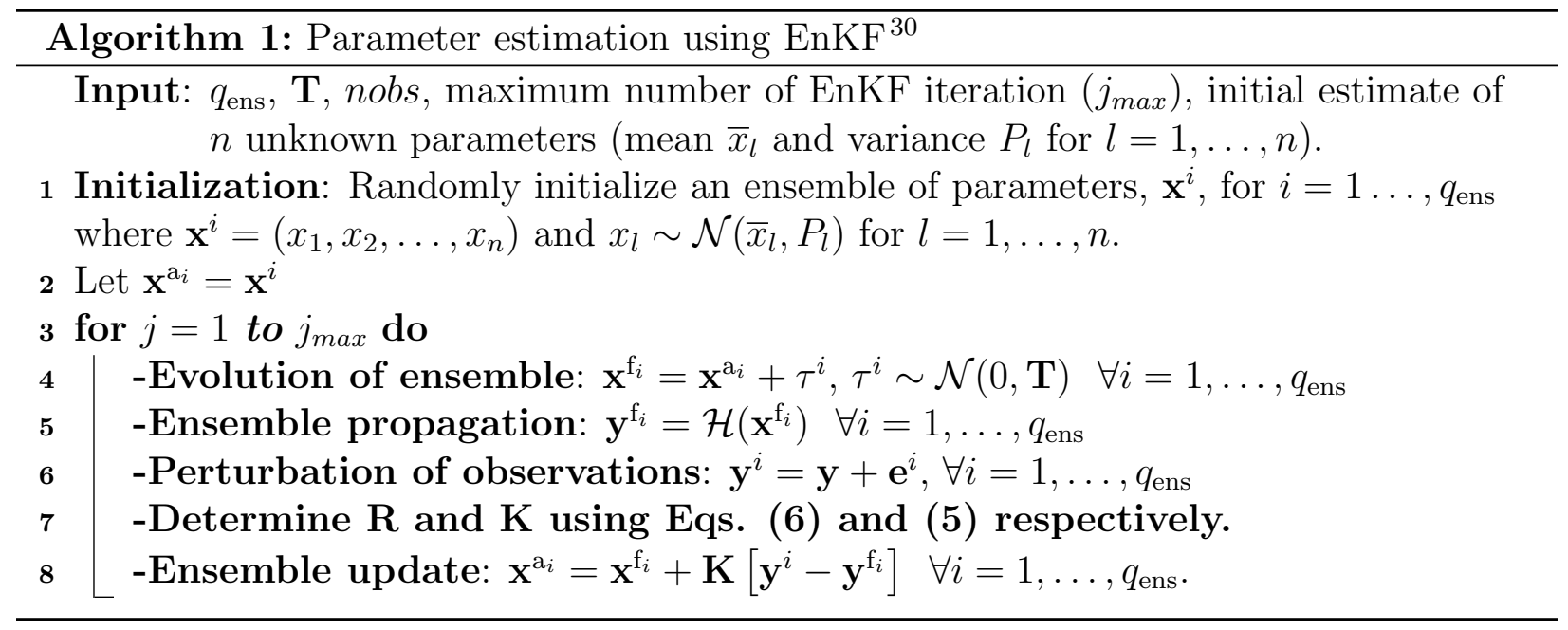

\subsection{Test cases}

The parameter estimation algorithm was tested using a complex arterial network with 33 arteries (Figure 2) consisting of the aorta, brachial, carotid and vertebral arteries as well as a complete $\mathrm{CoW}$ which was adapted from Alastruey et al. ${ }^{6}$. The objective was to identify (estimate) a set of model parameters within the physiological range, given measured values of arterial blood flow rate. The model parameters were limited to material properties and the terminal parameters (Windkessel parameters). Before the parameter estimation algorithm was tested with a patient-specific clinical data, a test case using synthetic data was carried out. This case where synthetic measurements were used in the data assimilation (observation obtained directly from the numerical model using the known model parameters) is presented in order to explore the capability of the EnKF algorithm. The test also aimed at finding if blood flow rates in the internal carotid arteries could be used as observations in the data assimilation to allow accurate estimates of model parameters. Another objective was to know for which network locations the pressure fluctuations are satisfactorily predicted.

\subsubsection{A test case using synthetic data}

We first describe the procedure for generating the synthetic data. The lengths, radii and the terminal boundary parameters (WK3) of the 33 arteries in Figure 2 were adapted

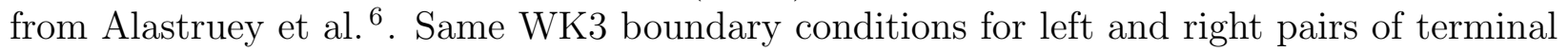
compartments were assumed. For instance, the terminal compartments \#10 and \#13 are assigned with the same WK3 boundary conditions. The product of Young's modulus and 


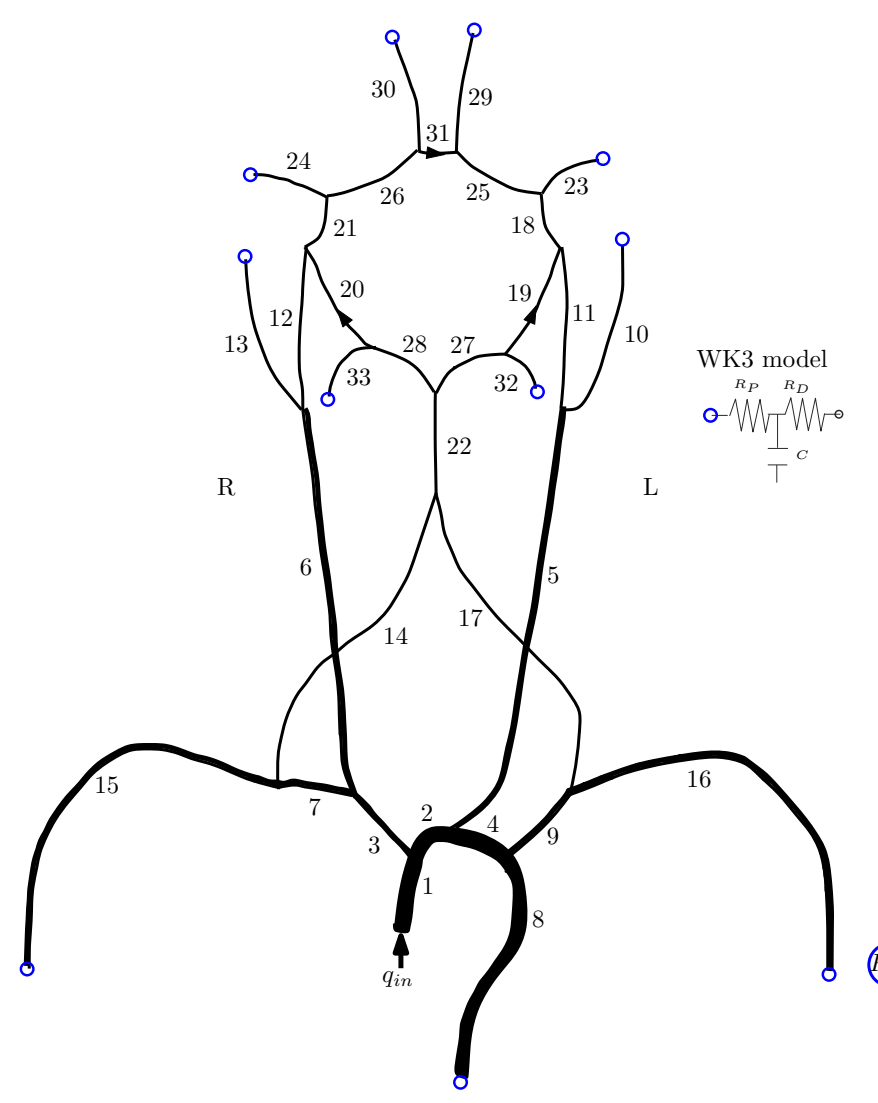

(a)

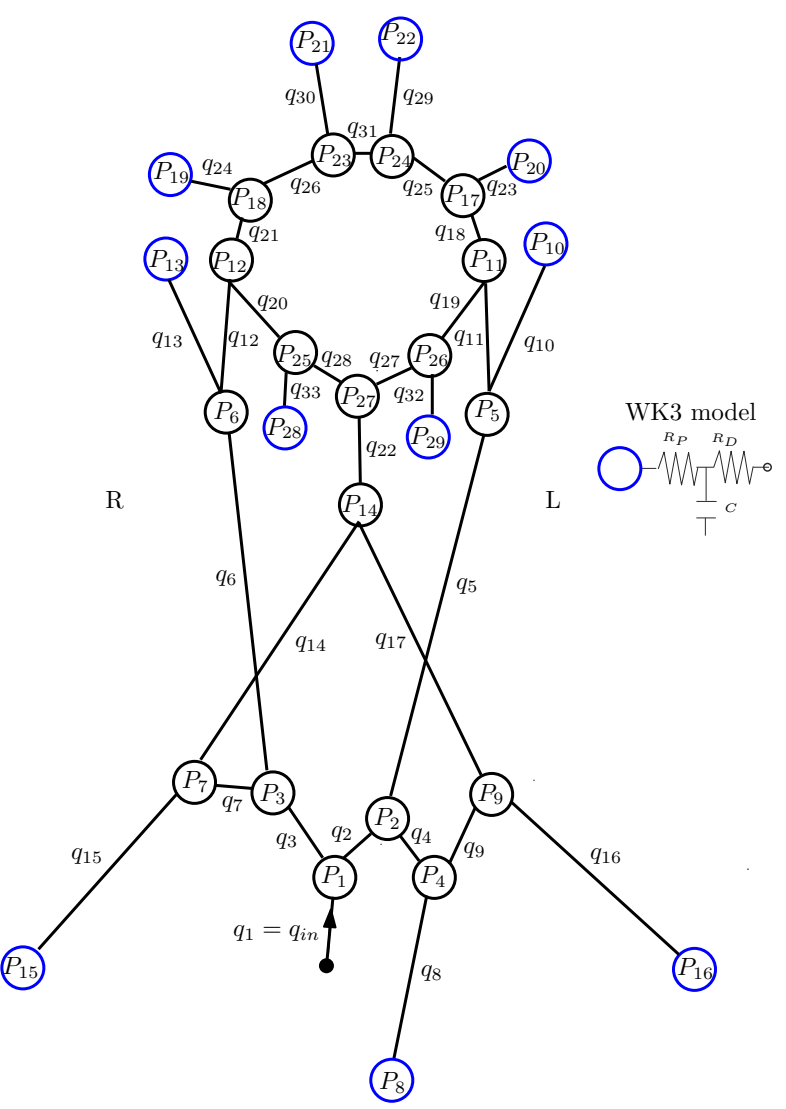

(b)

Figure 2: 2a The network ${ }^{6}$ of a one-dimensional blood flow model of the upper body arteries and of the circle of Willis. Thick lines indicate relative thickness of the arteries. Artery numbers corresponds to those in Table 2. Arrows indicate the direction of flow. (2b) The equivalent 0D compartment model. Flow rates are assigned the compartment numbers corresponding to those in Table 2. At the inlet (ascending aorta, compartment \#1), specific value of flow rates, $q_{i n}$, are imposed.

thickness of arteries was assumed to be given by an empirical formula $E h=r\left(k_{1} e^{k_{2} r}+k_{3}\right)^{38}$. The values of of the three constants were chosen as $k_{1}=5.0 \times 10^{7} \mathrm{~g} \cdot \mathrm{cm}^{-1} \mathrm{~s}^{-2}, k_{2}=-5.0 \mathrm{~cm}^{-1}$ and $k_{3}=1.0 \times 10^{5} \mathrm{~g} \cdot \mathrm{cm}^{-1} \mathrm{~s}^{-2}$. The three constants together with the WK3 parameters $R_{P_{i}}$, $R_{D_{i}}$ and $C_{i}$ where $i=8,13,15,24,30,33$ denotes the compartment number were referred as the target parameters. An inlet flow rate, $q_{i n}$, with period of $1 \mathrm{~s}$ was imposed at the proximal end of ascending aorta. For each cardiac cycle $q_{i n}$ in $\mathrm{ml} / \mathrm{s}$ was defined as ${ }^{6}$ :

$$
q_{\text {in }}(t)= \begin{cases}485 \sin (\pi t / \tau) & \text { if } t<\tau \\ 0 & \text { otherwise }\end{cases}
$$

where $\tau=0.3 \mathrm{~s}$. Blood rheological parameters were taken as: $\rho=1050 \mathrm{~kg} \cdot \mathrm{m}^{-3}$ and $\mu$ $=0.004 \mathrm{~Pa} \cdot \mathrm{s}$. Using the target parameters a forward simulation was executed using the 0D blood flow model. The blood flow rate values in the right internal artery (R-ICA: compartment \#12) and the left internal carotid artery (L-ICA: compartment \#11) were 
recorded.

Next, we describe the inverse hemodynamic problem. The 21 (target) parameters consisting of 6 proximal resistances, 6 distal resistances, 6 compliances and 3 constants defining the product $E h$ were estimated using the synthetic observations (blood flow rates in the two ICA's). The parameter estimation algorithm was initialised and executed using the following parameters:

1. Ensemble size: to test the sensitivity of the algorithm, different sizes of the ensemble $\left(q_{\text {ens }}=10\right.$ to $\left.q_{\text {ens }}=35\right)$ were chosen.

2. Initialisation: the 21 target parameters were randomly perturbed with increments of either $\pm 60 \%$ of the target values. These perturbed values were taken as the initial estimates. A larger perturbation (random increments of $\pm 100 \%$ of the target values) was also tried. It was noted that the results were similar than with $60 \%$. To restrict the assimilated parameters to positive values, all parameters were redefined as $x=x_{\text {ref }} 2^{\theta}$. $x_{\text {ref }}$ is the reference or initial guess of the parameter to be estimated and $\theta$ is the actual value used in the EnKF during the assimilation step. To initiate each assimilation cycle, an initial ensemble of parameters $\mathbf{x}^{i}$ for $i=1, \ldots, q_{\mathrm{ens}}$ was generated using the first-guess (initial estimate) value of the parameters $x_{\text {ref }}$ and $\theta$. For each $i^{\text {th }}$ member of the ensemble, $\mathbf{x}^{i}=\left(x_{1}, \ldots, x_{21}\right)$, where $x_{l}=x_{\text {ref }_{l}} 2^{\theta_{l}}$ and $\theta_{l}$ were random realizations from an $\mathcal{N}\left(0, \sigma_{\theta}^{2}\right)$ distribution for $l=1, \ldots, 21$ and $\sigma_{\theta}=0.5$. With each $\mathrm{x}^{i}$ the blood flow model was integrated for 10 cardiac cycles, and then the first assimilation was performed.

3. Number of observation: time series of blood flow rates were taken as observation at two locations $(n o b s=2)$ i.e. R-ICA and L-ICA, at every $0.02 \mathrm{~s}$ (50 per cardiac cycle).

4. Observation perturbation level: the observations $\mathbf{y}_{k}$ were perturbed by a random variable drawn from the observation error pdf $\sim \mathcal{N}\left(0, \sigma_{p}^{2}\right)$ where $\sigma_{p}$ represents the standard deviation. For the synthetic case, $\sigma_{p}$ was taken as $10 \%$ of the flow rate values of the R-ICA and L-ICA. For each $i^{\text {th }}$ member of an ensemble the perturbed observations were defined as $\mathbf{y}_{k}^{i}=\mathbf{y}_{k}+\mathbf{e}_{k}^{i}$, where $\mathbf{e}_{k}^{i}$ is a random number whose specific realization is obtained from a Gaussian distribution with mean zero and standard deviation $\sigma_{p}$.

5. Evolution of parameters: The evolution of each member of the estimated parameter ensemble was resembled by a random walk model. In the assimilation step, $\theta_{k+1}$ was defined as $\theta_{k+1}=\theta_{k}+\tau_{k}$ where $\tau_{k} \sim \mathcal{N}\left(0, \sigma_{\tau}^{2}\right)$ is a small Gaussian random perturbation. The updated parameters were allowed to vary continuously by choosing an arbitrary small value for $\tau_{k}$. Letting $\sigma_{\tau}^{2}<\kappa \sigma_{\theta}^{2}$, it was noted that for $0<\kappa<0.05$, improves the convergence rate of the estimation algorithm. Following this, in the rest of the paper we take $\tau_{k} \sim \mathcal{N}(0,0.001)$. This is an empirical construction and the idea motivating it comes from stochastic gradient methods with the aim of improving the search capacity of the algorithm by the introduction of small random perturbation to the descent direction. The final results are insensitive to the presence of this perturbation. The aim was to obtain an estimate of $\mathbf{x}^{i}$ by taking the limit as $\tau^{i} \rightarrow 0$. 
Figure 3 shows the time evolution of estimated parameters resulting from the EnKF for the six different ensemble size $\left(q_{\text {ens }}=10\right.$ to $\left.q_{\text {ens }}=35\right)$. For all cases, the filter converged in about 30 cardiac cycles and the estimated parameters converged to different values (non-unique). Parameter estimation using the EnKF method is found in this study to be very sensitive to the random realization of the initial ensemble and to the ensemble size. These results also indicate the increased difficulties when several parameters are estimated simultaneously.

Figure 4 shows the comparison between the target (blood flow waveform in the R-ICA and L-ICA) and blood flow model simulations (predictions) based on 21 estimated parameters using the six different ensemble size. There is a good agreement between the target and predicted flow rate waveform for the cases with $q_{\mathrm{ens}} \geq 25$. These results shows the interplay among influential parameters so that combinations of parameters with different errors can still result in a good fit of the model solution to the observations, rendering the solution to the parameter estimation problem non-unique.

In order to better compare the precision of the six cases, the percentage norm of the analysis error in the converged model solution relative to the norm of the target flow rate were calculated as:

$$
\frac{\left\|q_{a}-q_{\mathrm{tar}}\right\|}{\left\|q_{\mathrm{tar}}\right\|} \times 100 \%
$$

where $q_{a}$ denotes the converged solution (blood flow rate) when the model is simulated with the parameters estimated using different ensemble size, $q_{\mathrm{tar}}$ is the blood flow rate simulated using target parameters, and $\|\cdot\|$ is the $L_{2}$ norm. The analysis errors provide us with information on the closeness of different cases and also they give the difference between the predicted and the observed data. The percentage norm of the analysis error with the six cases are shown in the Figure 5. It is seen that the error decreases with the increase in the ensemble size. From this figure, the error does not decrease much after $q_{\text {ens }}=30$.

Finally, the predicted pressure fluctuations in the arteries were compared with the reference systolic blood pressure (SBP) and diastolic blood pressure (DBP). Here, 'reference' is defined as the pressure values (SBP and DBP) obtained by simulating the model with the target parameters. The predictions (with the six cases) and the reference pressure values are shown in Figure 6. The error in the predicted pressure values are the lowest $(<6 \%)$ for the cases with $q_{\mathrm{ens}} \geq 30$ as shown in Table 11. Pulse pressure (PP) $(\mathrm{SBP}-\mathrm{DBP})$ is likely to be the clinical measure of importance. The error in PP (see Table 1) are also the lowest $(<6 \%)$ for the cases with $q_{\mathrm{ens}} \geq 30$. The results show that the use of two flow rate measurements (as observations in data assimilation) at a relatively proximal locations (ICA's) in the network can lead to a set of model parameters for reliable prediction (with an error of less than 10\%) of pressure fluctuations in the cerebral arteries. Also, from the error analysis, as shown in the Figure 6 and the Table 1, an ensemble of size

$q_{\mathrm{ens}}=30$ seemed to be good enough to estimate 21 model parameters for the prediction of cerebral arterial pressure with an error of less than $10 \%$.

\subsubsection{A test case with patient specific PC-MRA\&MRI-based blood flow rates}

The patient-specific data used in the current study have been acquired at the Department of Neuroradiology at the Centre Hospitalier Régional Universitaire (CHRU), Montpellier, 

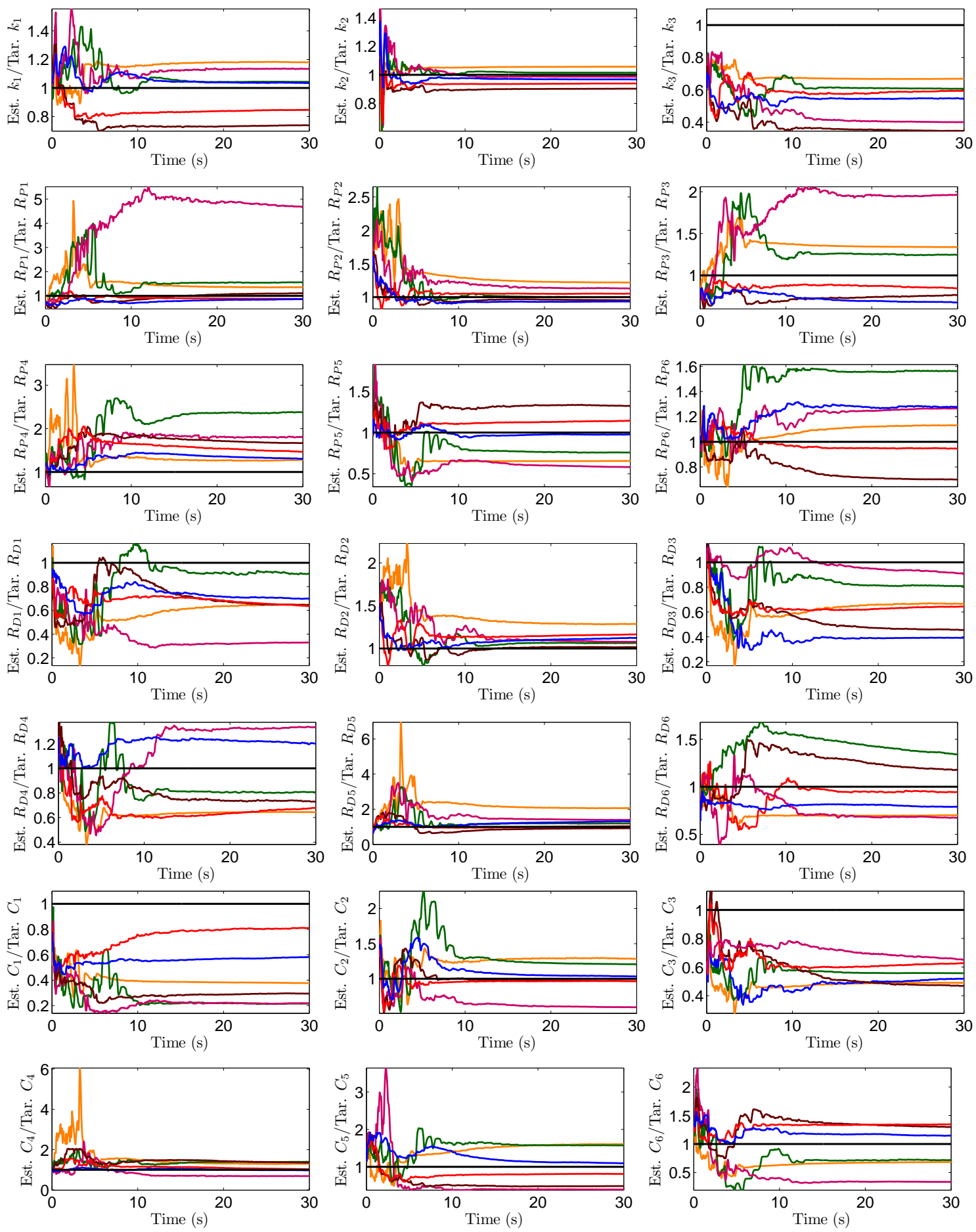

$$
\begin{gathered}
\text { - Target }=1,-q_{\mathrm{ens}}=10,-q_{\mathrm{ens}}=15,-q_{\mathrm{ens}}=20, \\
-q_{\mathrm{ens}}=25,-q_{\mathrm{ens}}=30,-q_{\mathrm{ens}}=35
\end{gathered}
$$

Figure 3: Estimated parameters with synthetic flow measurements using different ensemble size $\left(q_{\mathrm{ens}}=10\right.$ to $\left.q_{\mathrm{ens}}=35\right)$. The solid coloured line shows the estimated value over time (divided by the corresponding target values), so that 1 (horizontal black lines) corresponds to the target parameters. 

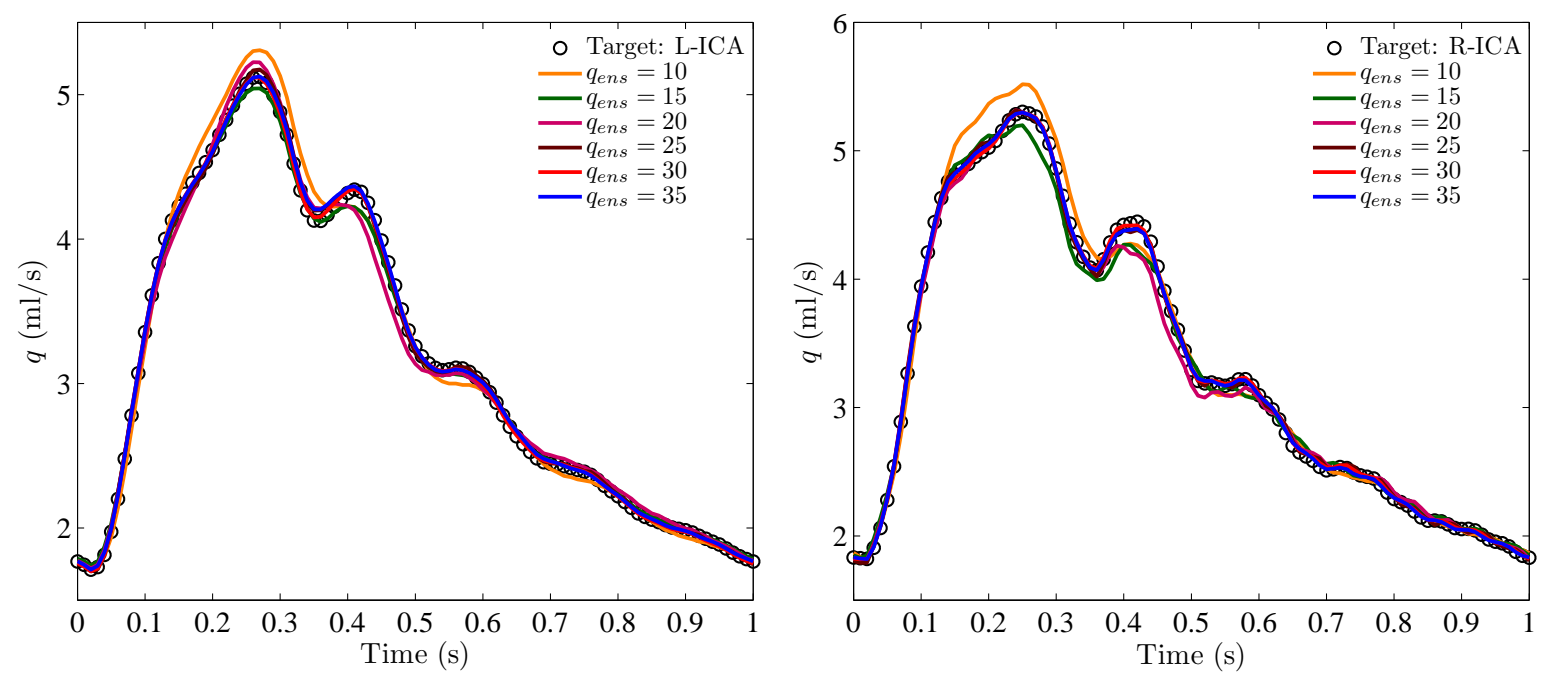

Figure 4: Comparison of the model simulated blood flow rate waveforms in R-ICA (right) and L-ICA (left) to the target (synthetic flow rate waveforms). The model simulations (predictions) were based on the 21 estimated parameters using the six different ensemble size.

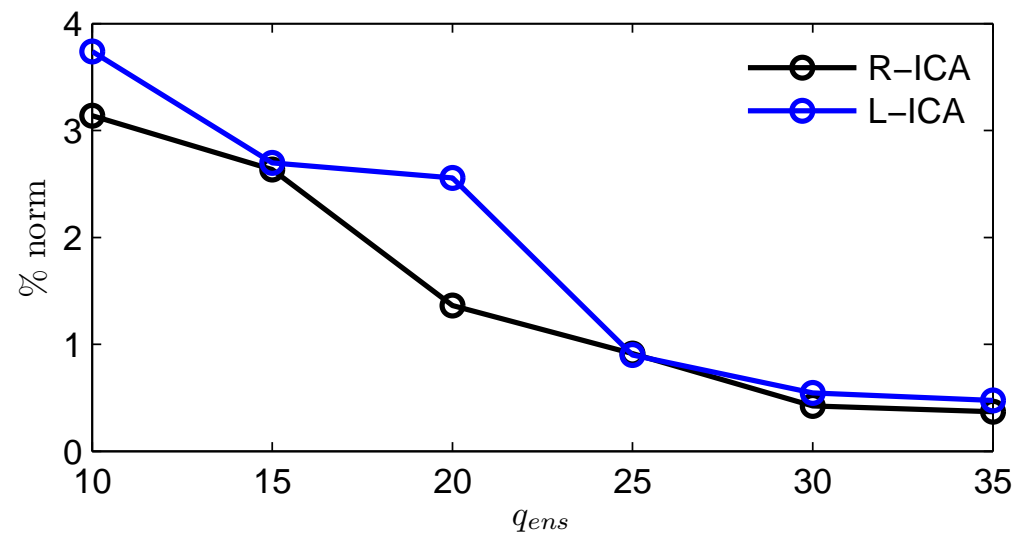

Figure 5: Percentage norm of the analysis error versus ensemble size. 


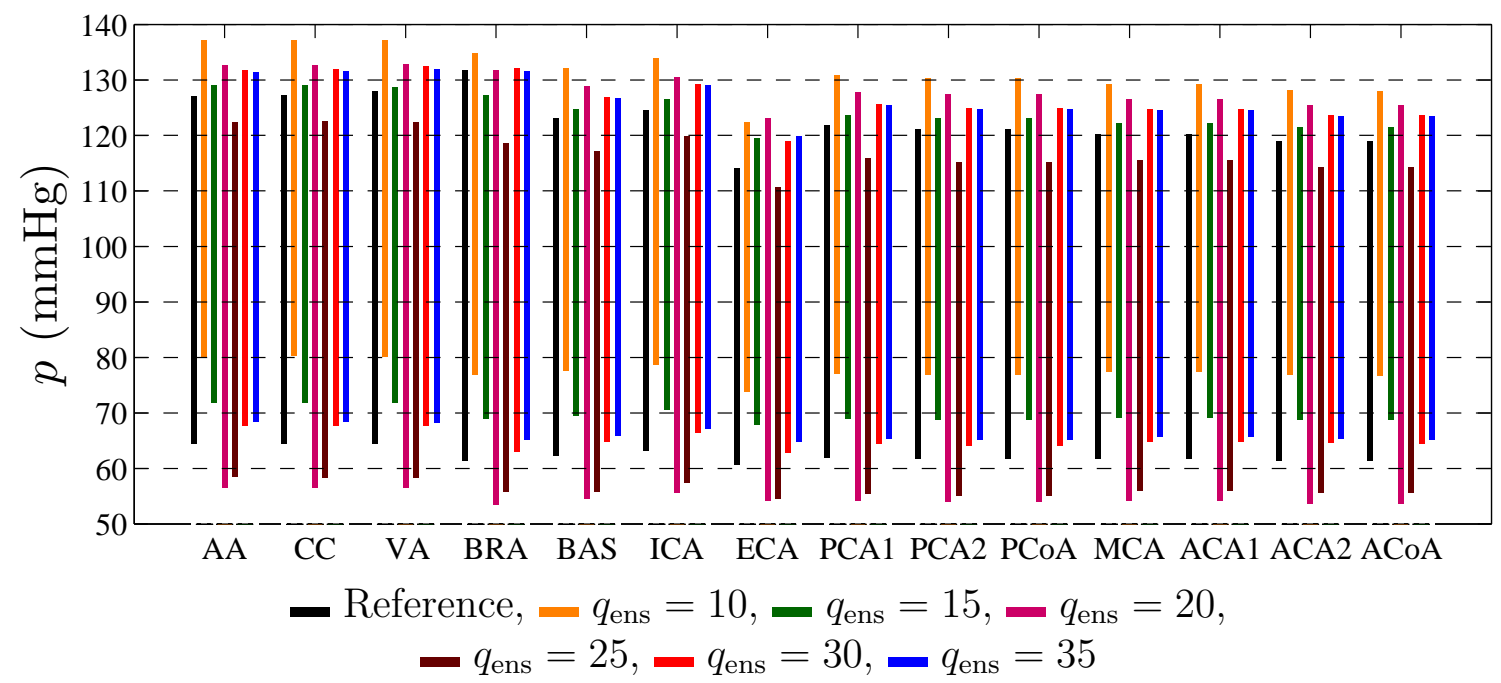

Figure 6: The predicted pressure fluctuations in the arteries compared with the reference systolic blood pressure and diastolic blood pressure. The predictions were based on 21 estimated parameters using the six different ensemble size. 'Reference' pressure values were obtained by simulating the model with the target parameters and are shown in solid black lines. The names of the arteries are written using acronyms which correspond to those in Table 2 .

Table 1: The maximum percentage error in (i) the predicted pressure fluctuations (diastolic and systolic) and (ii) the pulse pressure (PP) in the arteries. The predictions were based on 21 estimated parameters using the six different ensemble size.

\begin{tabular}{lccc}
\hline Case & $\begin{array}{c}\text { Error in the predicted pressure values } \\
\text { diastolic }\end{array}$ & $\begin{array}{c}\text { Error in the pulse } \\
\text { pressure }\end{array}$ \\
\hline$q_{\text {ens }}=10$ & $<26 \%$ & $<8 \%$ & \\
$q_{\text {ens }}=15$ & $<13 \%$ & $<5 \%$ & $<18 \%$ \\
$q_{\text {ens }}=20$ & $<13 \%$ & $<8 \%$ & $<18 \%$ \\
$q_{\text {ens }}=25$ & $<11 \%$ & $<10 \%$ & $<25 \%$ \\
$q_{\text {ens }}=30$ & $<6 \%$ & $<5 \%$ & $<10 \%$ \\
$q_{\text {ens }}=35$ & $<6 \%$ & $<5 \%$ & $<6 \%$ \\
\hline
\end{tabular}

France.

Arterial systolic and diastolic blood pressures at rest of the patient were measured before and after image acquisition using a brachial automatic sphygmomanometer (Maglife, Schiller Medical). The systolic and diastolic values were $125 \mathrm{mmHg}$ and $72 \mathrm{mmHg}$ in the right brachial artery and $115 \mathrm{mmHg}$ and $72 \mathrm{mmHg}$ in the left brachial artery. 2D phase-contrast imaging was performed on a Siemens 3T Skyra MR Scanner. The ascending aorta and the internal carotid arteries (right and left ICA's) were considered for the analysis of blood flow rates. More precisely, 2D Fast cine PC-MRI pulse sequence (one $5 \mathrm{~mm}$ slice perpendicular to the arteries) with retrospective peripheral gating, and 32 frames covering the entire cardiac cycle were acquired. The imaging parameters for ICA's 
were a velocity encoding sensitivity (Venc) of $80 \mathrm{~cm} \cdot \mathrm{s}^{-1}$, a repetition time (TR) of $28.86 \mathrm{~ms}$, an echo time (TE) of $8.79 \mathrm{~ms}$, a flip angle of $15^{\circ}$, and a voxel size of $0.53 \mathrm{~mm} \times 0.53 \mathrm{~mm} \times 5.0 \mathrm{~mm}$.

Figure 7 shows one pair of the acquired images (magnitude and phase contrast image) for ICA's. For the flow rate analysis, the Bio Flow Image software (http://www.tidam.fr/) was employed. For each of the arteries, a region of interest (ROI) was segmented with its lumen size defined by thresholding. For the ascending aorta, the imaging parameters
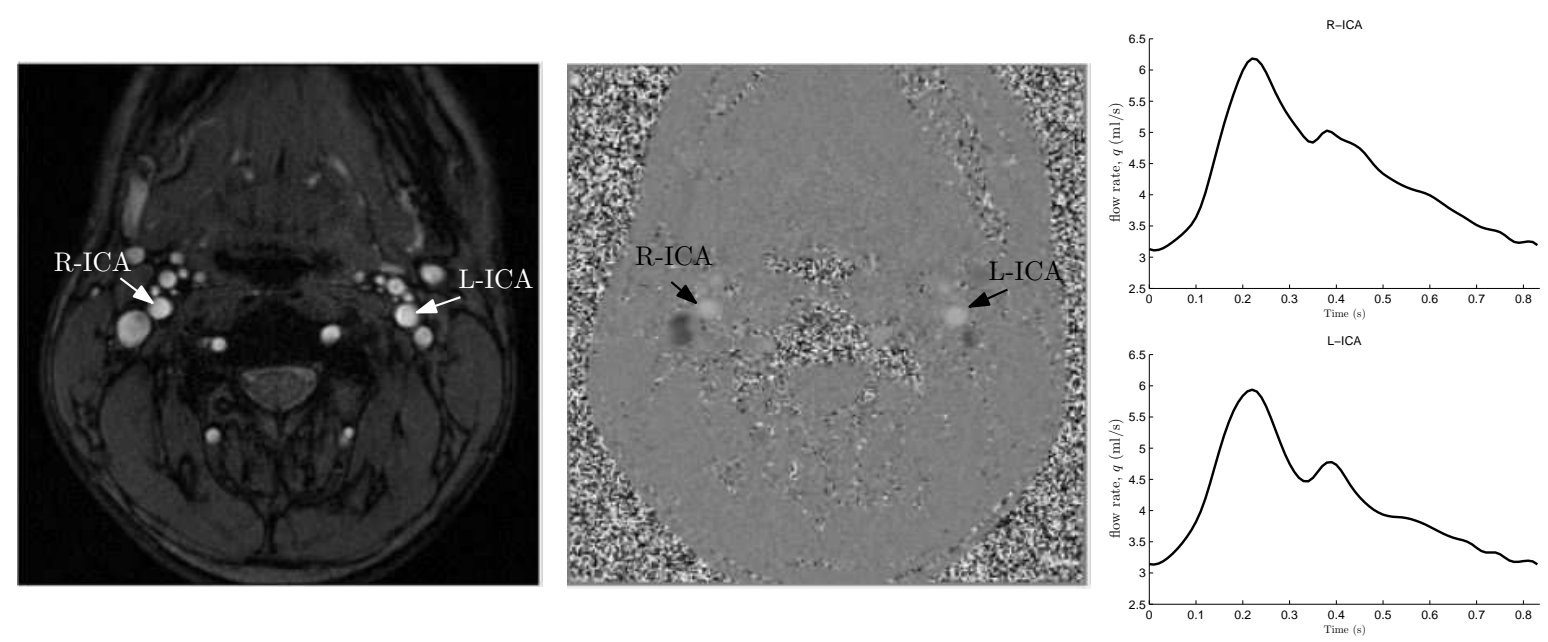

Figure 7: PC-MRI of the patient-specific internal carotid arteries (right and left) showing the blood flow through one of the selected slices. On the left is the magnitude image and in the centre is the phase contrast image with Venc setting of $80 \mathrm{~cm} \cdot \mathrm{s}^{-1}$. The instantaneous blood flow rate values, $q(t)$ are acquired at each time frame and is plotted against time for one cardiac cycle as shown on the right.

were: Venc $=200 \mathrm{~cm} \cdot \mathrm{s}^{-1}, \mathrm{TR}=28.72 \mathrm{~ms}, \mathrm{TE}=8.79 \mathrm{~ms}$, flip angle $15^{\circ}$ and a voxel size $0.57 \mathrm{~mm} \times 0.57 \mathrm{~mm} \times 5.0 \mathrm{~mm}$. Figure 8 shows one pair of the magnitude and phase contrast image acquired for the ascending aorta. The corresponding blood flow rate is also shown in the same figure.

A 3D Time of Flight magnetic resonance angiography (3D-TOF-MRA) of the circle of Willis was obtained with the parameters: acquired voxel $0.31 \times 0.31 \times 0.55 \mathrm{~mm}, 28$ slices, $\mathrm{TR}=21.0 \mathrm{~ms}, \mathrm{TE}=3.49 \mathrm{~ms}$ and flip angle of $28^{\circ}$.

The 3D model (and morphology) of the circle of Willis (see Figure 9) was determined through segmentation of the TOF MRA using RadiAnt DICOM Viewer software (http: //www.radiantviewer.com/). The geometric measurements of lengths and radii of CoW's blood vessel were measured from MRA and are shown in Table 2. The carotid vascular tree could not be obtained because this acquisition requires the injection of contrast agent which is impossible to achieve on healthy volunteers. The missing geometry of other arteries of the full network (Figure 2b) was obtained from average data reported in the literature ${ }^{6 / 7}$. 

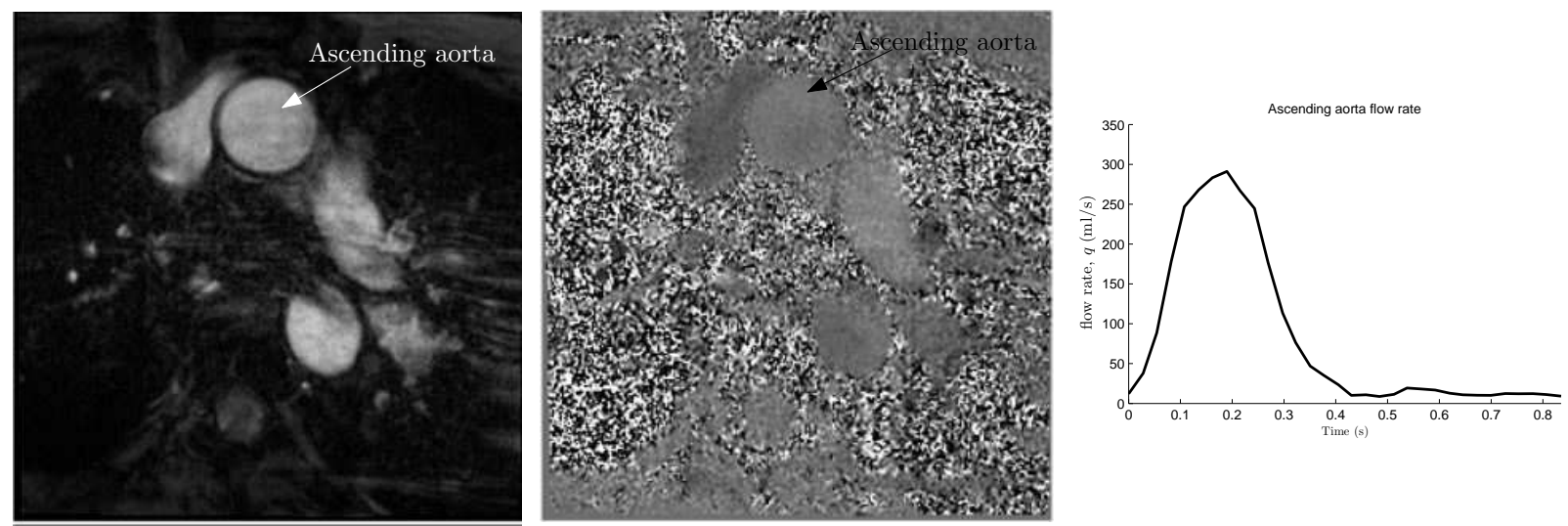

Figure 8: PC-MRI of the patient-specific ascending aorta showing the blood flow through one of the selected slices. On the left is the magnitude image and in the centre is the phase contrast image with Venc setting of $200 \mathrm{~cm} \cdot \mathrm{s}^{-1}$. The instantaneous blood flow rate values, $q(t)$ were acquired at each time frame and is plotted against time for one cardiac cycle as shown on the right.
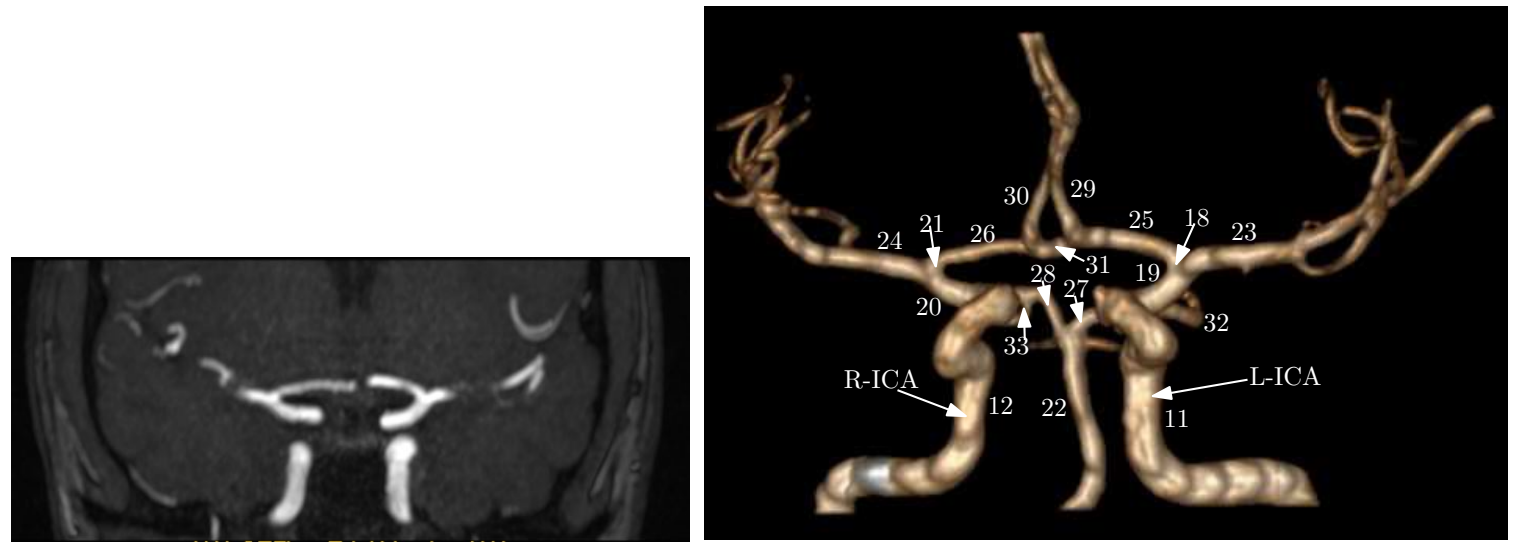

Figure 9: A TOF MRI scan (left) and the resulting segmented 3D model of the complete circle of Willis (right) for patient-specific case. The numbers on segmented model correspond to the ID's of the arterial segments in Table 2 .

The inverse hemodynamic problem was set up as follows: using data assimilation, arterial stiffness and WK3 model boundary parameters were sought for the network as shown in Figure 2b. The acquired flow rate waveform for the right internal carotid (R-ICA) was used as observations during EnKF assimilation steps in the parameter estimation problem. The flow rate waveforms for the left internal carotid (L-ICA) was used in a posteriori validation process. For the forward simulation during the data assimilation the 0D compartment model was employed. At the inlet (ascending aorta, compartment \#1 in Figure 2b), specific values of flow rates, $q_{i n}$, were imposed as measured by PC-MRI (see Figure 8). Blood rheological parameters were taken as: $\rho=1050 \mathrm{~kg} \cdot \mathrm{m}^{-3}$ and $\mu=0.004 \mathrm{~Pa} \cdot \mathrm{s}$. Although not detailed in the paper for sake of conciseness, several assimilation scenarii were tested to investigate the robustness of the whole procedure.

The results shown in the remaining of the paper were obtained under the following 
Table 2: Geometric parameters corresponding to arterial segments (and compartments) in Figures 2 and 9 measured from MRI. The missing geometry (marked with an asterisk) of larger arteries was adopted from the average data in the literature 6 . $\mathrm{R}$ indicates right; $\mathrm{L}$ left.

\begin{tabular}{|c|c|c|c|c|c|c|c|}
\hline id & Name & $l(\mathrm{~cm})$ & $r(\mathrm{~cm})$ & id & Name & $l(\mathrm{~cm})$ & $r(\mathrm{~cm})$ \\
\hline 1 & Ascending aorta (AA) & $4.00^{*}$ & $1.200^{*}$ & 18 & L.internal carotid II & 0.50 & 0.200 \\
\hline 2 & Brachiocephalic & $2.00^{*}$ & $1.120^{*}$ & 19 & L.post. comm. artery (PCoA) & 1.20 & 0.075 \\
\hline 3 & Aortic arch II & $3.40^{*}$ & $0.620^{*}$ & 20 & R.post. comm. artery (PCoA) & 1.20 & 0.075 \\
\hline 4 & Aortic arch II & $3.90^{*}$ & $1.070^{*}$ & 21 & R.internal carotid II & 0.50 & 0.200 \\
\hline 5 & L.common carotid (L.CC) & $20.8^{*}$ & $0.250^{*}$ & 22 & Basilar (BAS) & 2.70 & 0.150 \\
\hline 6 & R.common carotid (R.CC) & $17.7^{*}$ & $0.250^{*}$ & 23 & L.middle cerebral artery (MCA) & 11.9 & 0.143 \\
\hline 7 & R.subclavian & $3.40^{*}$ & $0.423^{*}$ & 24 & R.middle cerebral artery (MCA) & 11.9 & 0.143 \\
\hline 8 & Thoracic aorta & $15.6^{*}$ & $0.999^{*}$ & 25 & L.anterior cerebral artery A1 (ACA, A1) & 1.20 & 0.117 \\
\hline 9 & L.subclavian & $3.40^{*}$ & $0.423^{*}$ & 26 & R.anterior cerebral artery A1 (ACA, A1) & 1.20 & 0.117 \\
\hline 10 & L.external carotid (L.ECA) & $17.7^{*}$ & $0.150^{*}$ & 27 & L.post. cerebral artery P1 (PCA, P1) & 0.56 & 0.110 \\
\hline 11 & L.internal carotid I (L.ICA) & $17.7^{*}$ & 0.200 & 28 & R.post. cerebral artery P1 (PCA, P1) & 0.56 & 0.110 \\
\hline 12 & R.internal carotid I (R.ICA) & $17.7^{*}$ & 0.200 & 29 & L.anterior cerebral artery A2 (ACA, A2) & 10.3 & 0.120 \\
\hline 13 & R.external carotid (R.ECA) & $17.7^{*}$ & $0.150^{*}$ & 30 & R.anterior cerebral artery A2 (ACA, A2) & 10.3 & 0.120 \\
\hline 14 & R.vertebral (R.VA) & $14.8^{*}$ & $0.136^{*}$ & 31 & Anterior comm. artery (ACoA) & 0.30 & 0.074 \\
\hline 15 & R.brachial (R.BRA) & $42.2^{*}$ & $0.403^{*}$ & 32 & L.post. cerebral artery P2 (PCA, P2) & 8.50 & 0.100 \\
\hline 16 & L.brachial (L.BRA) & $42.2^{*}$ & $0.403^{*}$ & 33 & R.post. cerebral artery P2 (PCA, P2) & 8.50 & 0.100 \\
\hline 17 & L.vertebral (L.VA) & $14.8^{*}$ & $0.136^{*}$ & & & & \\
\hline
\end{tabular}

assumptions on the unknown model parameters:

The patient-specific characterisation of the 0D blood flow model represented by Equation (2.1) is achieved through the model parameters $R, L, C$ and the WK3 boundary condition parameters. The parameters $R, L$ and $C$ require the measurements of the length and the radius of each arterial segment in the network, and these are measured from MRA. In addition, the parameter $C$ depends on the arterial stiffness that is determined by the Young's modulus $(E)$ and the wall thickness $(h)$. $E$ cannot be determined directly using imaging techniques and need further computations (or estimation). $C$ is linear in $E h$ and this is the unknown quantity to recover by data assimilation. The product of Young's modulus and thickness of arteries was assumed to be given by an empirical formula $E h=r\left(k_{1} e^{k_{2} r}+k_{3}\right)^{38}$ where the known radius $(r)$ came from MRA. An estimation of the product $E h$ was found by seeking an estimation of the unknown constants with their initial guesses as $k_{1}=2.0 \times$ $10^{7} \mathrm{~g} \cdot \mathrm{cm}^{-1} \mathrm{~s}^{-2}, k_{2}=-22.0 \mathrm{~cm}^{-1}$ and $k_{3}=8.0 \times 10^{5} \mathrm{~g} \cdot \mathrm{cm}^{-1} \mathrm{~s}^{-2}$. The same assumption on WK3 boundary conditions for left and right pairs of terminal compartments as in section 2.3.1 was taken here, and the parameters $R_{P_{i}}, R_{D_{i}}$ and $C_{i}$ where $i=8,13,15,24,30,33$ denotes the compartment number were also considered as unknown model parameters. Thus, the same 21 parameters as in the section 2.3.1 are estimated. Furthermore, for arteries with $r<0.2 \mathrm{~cm}$, the inertial effect was ignored ${ }^{39}$ in the 0D compartment model during the forward simulation.

As in the section 2.3.1, the parameter estimation algorithm was initialised and executed using the following parameters:

1. Ensemble size: from the findings of the synthetic case, $q_{\mathrm{ens}}=30$ appears to be a suitable choice for the ensemble size.

2. Initialisation: The initial estimates for proximal resistances and compliances were taken

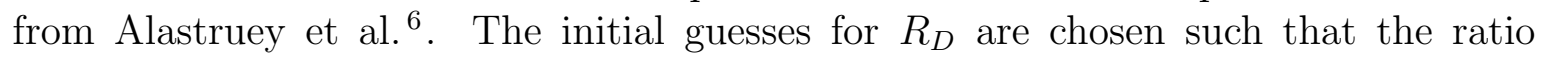


$R_{P} /\left(R_{P}+R_{D}\right)=0.2$, i.e $R_{D}=4 R_{P}$ 40. The rest of the procedure for reparametrization introducing $\theta$ and generation of the initial ensemble was as described in the section 2.3 .1 ,

3. Number of observation: time series of blood flow rates from R-ICA $(n o b s=1)$ were taken as observations at every $0.04175 \mathrm{~s}$ (20 EnKF iterations per cardiac cycle).

4. Observation perturbation level: it has been reported that the blood flow rates measured by $3 \mathrm{~T}$ phase contrast MRI is accurate within $10-15 \%$ of the true flow rates $\underline{4143}$. Also, using in vitro phantom studies, Tang et al. $\underline{44}$ demonstrated that the error in volume flow rate measurements obtained with phase-contrast methods would be $<10 \%$ if the ratio between the voxel length and vessel radius was less than 0.5. For the current case, $\sigma_{p}$ was taken as $10 \%$ of the flow rate values of the R-ICA. The perturbed observations for the $i^{\text {th }}$ member of the ensemble were defined as $\mathbf{y}_{k}^{i}=\mathbf{y}_{k}+\mathbf{e}_{k}^{i}$, where $\mathbf{e}_{k}^{i}$ is a random number whose specific realization is obtained from a Gaussian distribution with mean zero and standard deviation $\sigma_{p}$.

5. Evolution of parameters: the procedure was the same as described in the section 2.3.1.

The parameter estimation algorithm was executed for $60 \mathrm{~s}$ (approximately 72 cardiac cycles). The above scenario for the parameter estimation was referred as scenario A. The results from this scenario were obtained by assimilating 21 parameters and running a physical model based on Ordinary Differential Equations like Eqs. (1)-(2).

A parameter estimation sensitivity analysis was performed using two more scenarii. The objective of this section was to analyse the relative sensitivity of the estimated parameters with respect to the observation location and their numbers nobs. It is recalled that the previous scenario (scenario A), uses nobs $=1$ and estimates 21 parameters using the flow rate waveform for the R-ICA as observations. To see the impact of a change in the target observations, the acquired flow rate waveform for the left internal carotid (L-ICA) was considered as the target during EnKF assimilation steps while the a posteriori validation process considers the flow rate waveforms in the right internal carotid (R-ICA). This was called scenario B. Also, to see the impact of a change in the number of observations, both available observations were considered as target which means nobs $=2$. This was called scenario C. All other parameters and settings were same as for the scenario A. Table 3 provides a summary of the three scenarii.

Table 3: Main characteristics of the three scenarii considered.

\begin{tabular}{|c|c|c|c|}
\hline Scenario & Location of observation(s) & \multicolumn{2}{|c|}{ Quantity used for a poseriori assessment } \\
\hline $\mathrm{A}$ & R-ICA & $\begin{array}{l}\text { flow rate waveform } \\
\text { for the L-ICA }\end{array}$ & \multirow{3}{*}{$\begin{aligned} & \text { brachial artery pressure: } \\
& \text { - right: } \text { systolic } 125 \mathrm{mmHg} \\
& \text { diastolic } 72 \mathrm{mmHg} \\
& \text { - left: } \text { systolic } 115 \mathrm{mmHg} \\
& \text { diastolic } 72 \mathrm{mmHg}\end{aligned}$} \\
\hline $\mathrm{B}$ & L-ICA & $\begin{array}{l}\text { flow rate waveform } \\
\text { for the } \mathrm{R}-\mathrm{ICA}\end{array}$ & \\
\hline $\mathrm{C}$ & $\begin{array}{l}\text { R-ICA and } \\
\text { L-ICA }\end{array}$ & - & \\
\hline
\end{tabular}




\section{Results}

Figure 10 shows the time evolution of estimated parameters with the three scenarii: scenario A (in blue), scenario B (in red), and scenario C (in black). With all scenarii, the filter converged in about 72 cardiac cycles and the estimated parameters converged to different values for the three scenarii. The final estimates of the 21 parameters with their associated uncertainties using the three scenarii are summarised in Table 4 . What is important to note, however, is that the three scenarii lead to very similar results in terms of flowrate. This is illustrated in Figure 11, which shows the comparison between the observed (clinically measured blood flow rates using MRI) and blood flow model simulations (predictions) based on 21 estimated parameters using the three scenarii. Note that the red curve in Figure $11 \mathrm{a}$ and the blue curve in Figure $11 \mathrm{~b}$ correspond to the a posteriori validation blood flow waveforms with the scenario A and scenario B respectively. From the results, the comparison between the assimilated OD model and in vivo data (MRI) is fair. It is observed that secondary peaks in the flow rate waveforms are reproduced with the model. Also, the 0D compartment blood flow model and the EnKF parameter estimation algorithm are seen to be compatible with nobs of more than 1 . With all the three scenarii, the model simulated waveforms have an error of less than $5 \%$ in the diastolic and systolic flow rate.

Table 5 summarizes the cardiac cycle-averaged (mean) and maximum (peak) volumetric flow rates measured in the ICA's using MRI and simulated values using the model with estimated parameters. All mean, peak (systolic), and diastolic flow rates measurements and model outputs (for the three scenarii) differ by less than $6 \%$. Additionally, the mean and peak values are compared with and found to be within the reported range in Ford et al. $\frac{45}{\text {. }}$ Furthermore, it is observed that errors (see Table 5) in peak and mean values of the blood flow rate in the ICA's are least with the scenario $\mathrm{C}$ when compared with the MRI data.

The systolic blood pressure (SBP) and diastolic blood pressure (DBP) in major arteries constituting the circle of Willis, vertebral arteries, and some larger arteries are predicted using the scenarii A, B and C. The predicted pressure values (SBP and DBP) are shown in Figure 12. With all the scenarii the predicted pressures in the arteries constituting the

CoW are within the physiological values of a healthy patient 4 . With the scenario $\mathrm{B}$, slightly higher SBP is predicted in all the arteries. Despite these little differences, one can, however, conclude that the three scenarii give nearly the same pressure level predictions in the different arteries.

\section{Discussion}

The objective of the test case is to identify a set of model parameters from measured values (observations) of flow rate. Towards this end, a 0D compartment model is found to be much faster than a classical 1D PDE-based blood flow model yet providing very similar results in terms of blood pressure and flow rate signals (not shown). In determining the Kalman gain, the EnKF uses estimates that are based on the ensemble size and the ensemble members. After a synthetic case analysis, we have noticed that an ensemble size of 30 is suitable for our problem involving 21 uncertain variables. The in vivo inversion shows good agreement between the measured flow rate from MRI and the outcome of the assimilated compartment 

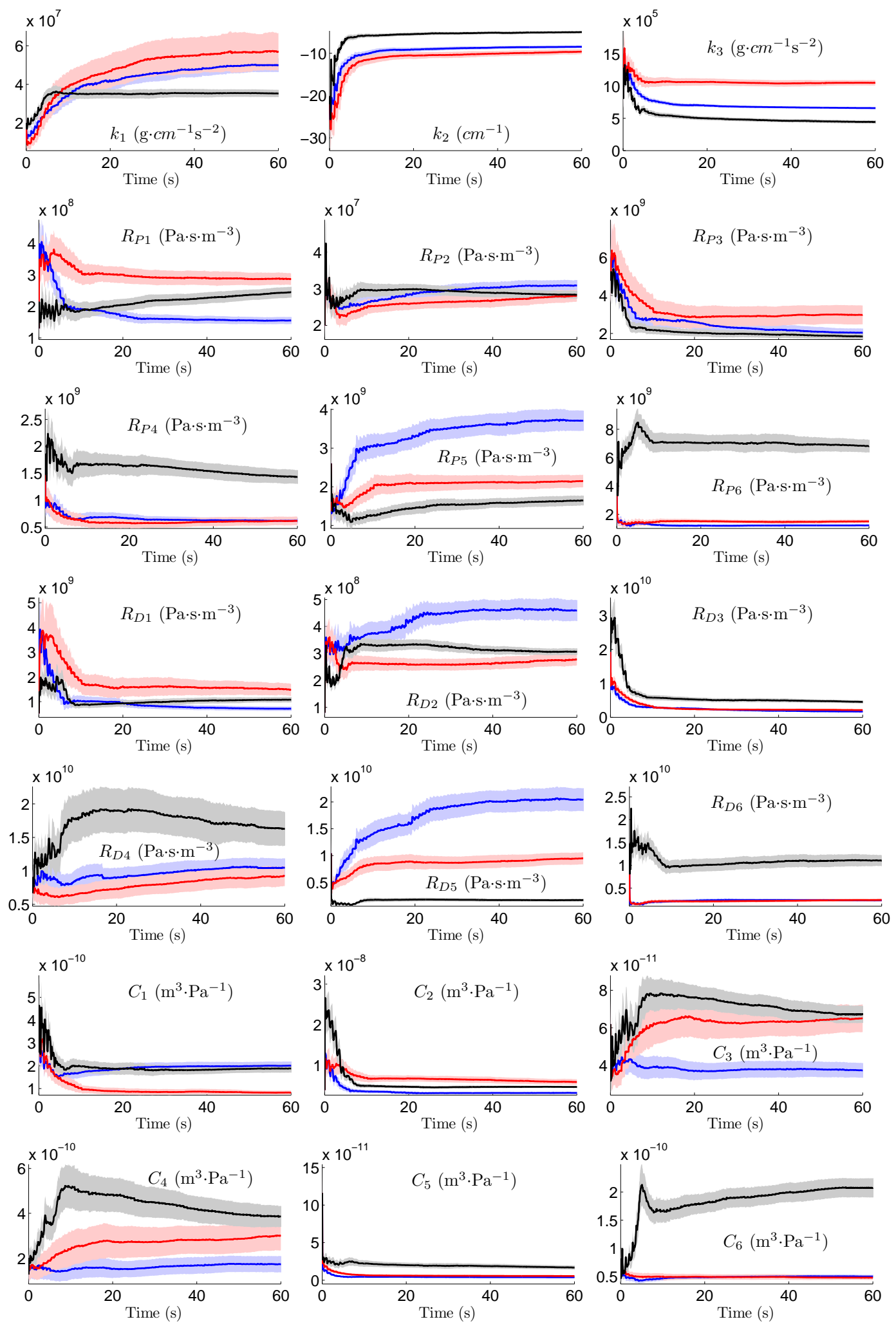

Figure 10: The patient-specific fitting. Time evolution of estimated parameters with the three scenarii: scenario A (in blue), scenario B (in red), and scenario C (in black). The shaded areas represent the standard deviation around the ensemble mean values (solid lines). 
Table 4: Estimated parameters (with associated errors) for the patient specific fitting with the three scenarii: the scenarii $\mathrm{A}, \mathrm{B}$ and $\mathrm{C}$. The values of constants $k_{1}, k_{2}$, and $k_{3}$ are in $\times 10^{7} \mathrm{~g} \cdot \mathrm{cm}^{-1} \mathrm{~s}^{-2}, \mathrm{~cm}^{-1}$, and $\times 10^{5} \mathrm{~g} \cdot \mathrm{cm}^{-1} \mathrm{~s}^{-2}$ respectively. The proximal $\left(R_{P}\right)$ and distal $\left(R_{D}\right)$ resistances are in $\times 10^{9} \mathrm{~Pa} \cdot \mathrm{s} \cdot \mathrm{m}^{-3}$ and the compliance $(C)$ are in $\times 10^{-10} \mathrm{~m}^{3} \cdot \mathrm{Pa}^{-1}$.

\begin{tabular}{lcccc}
\hline Parameter & Initial guess & \multicolumn{3}{c}{ Final EnKF estimate \pm error } \\
& & scenario A & scenario B & scenario C \\
\hline$k_{1}$ & 2.00 & $4.99 \pm 0.36$ & $5.70 \pm 0.96$ & $3.53 \pm 0.19$ \\
$k_{2}$ & -22.0 & $-8.47 \pm 0.32$ & $-9.62 \pm 0.60$ & $-5.00 \pm 0.27$ \\
$k_{3}$ & 8.5 & $6.60 \pm 0.20$ & $10.52 \pm 0.41$ & $4.44 \pm 0.25$ \\
$R_{P 8}$ & 0.02 & $0.031 \pm 0.002$ & $0.03 \pm 0.002$ & $0.028 \pm 0.001$ \\
$R_{P 13}$ & 1.67 & $0.62 \pm 0.05$ & $0.62 \pm 0.08$ & $1.43 \pm 0.13$ \\
$R_{P 15}$ & 0.13 & $0.15 \pm 0.01$ & $0.29 \pm 0.02$ & $0.24 \pm 0.02$ \\
$R_{P 24}$ & 2.61 & $3.71 \pm 0.26$ & $2.15 \pm 0.16$ & $1.64 \pm 0.13$ \\
$R_{P 30}$ & 3.70 & $1.23 \pm 0.07$ & $1.51 \pm 0.12$ & $6.81 \pm 0.44$ \\
$R_{P 33}$ & 4.8 & $2.04 \pm 0.22$ & $2.98 \pm 0.49$ & $1.84 \pm 0.15$ \\
$R_{D 8}$ & 0.08 & $0.46 \pm 0.04$ & $0.28 \pm 0.001$ & $0.31 \pm 0.001$ \\
$R_{D 13}$ & 6.68 & $10.44 \pm 1.40$ & $9.23 \pm 0.20$ & $16.19 \pm 0.22$ \\
$R_{D 15}$ & 0.52 & $0.71 \pm 0.09$ & $1.48 \pm 0.016$ & $1.00 \pm 0.01$ \\
$R_{D 24}$ & 10.44 & $20.42 \pm 2.13$ & $9.48 \pm 0.08$ & $1.69 \pm 0.02$ \\
$R_{D 30}$ & 14.80 & $2.33 \pm 0.33$ & $2.40 \pm 0.03$ & $11.03 \pm 0.08$ \\
$R_{D 33}$ & 19.32 & $1.70 \pm 0.15$ & $2.13 \pm 0.04$ & $4.53 \pm 0.04$ \\
$C_{8}$ & 38.78 & $31.90 \pm 2.47$ & $58.78 \pm 6.86$ & $46.44 \pm 2.54$ \\
$C_{13}$ & 1.27 & $1.73 \pm 0.36$ & $3.00 \pm 0.65$ & $3.86 \pm 0.47$ \\
$C_{15}$ & 2.58 & $2.00 \pm 0.18$ & $0.82 \pm 0.10$ & $1.88 \pm 0.18$ \\
$C_{24}$ & 1.16 & $0.04 \pm 0.004$ & $0.06 \pm 0.006$ & $0.17 \pm 0.03$ \\
$C_{30}$ & 0.82 & $0.49 \pm 0.03$ & $0.48 \pm 0.04$ & $2.12 \pm 0.17$ \\
$C_{33}$ & 0.62 & $0.37 \pm 0.04$ & $0.65 \pm 0.07$ & $0.66 \pm 0.05$ \\
\hline & & & & \\
\hline
\end{tabular}

Table 5: Sensitivity analysis: comparison of the cardiac cycle-averaged (mean) and maximum (peak) volumetric flow rates of model simulated values with scenarii A, B and C to measured values using MRI in the ICA's. The percentage difference between the model results and the MRI data are stated in bold in parenthesis. The model results are also compared with the reported values in Ford et al. $\stackrel{45}{\text {. }}$

\begin{tabular}{|c|c|c|c|c|c|c|c|c|c|c|}
\hline \multirow[t]{3}{*}{ ICA } & \multicolumn{5}{|c|}{ Maximum (peak) flow rate $(\mathrm{ml} / \mathrm{s})$} & \multicolumn{5}{|c|}{ Mean flow rate $(\mathrm{ml} / \mathrm{s})$} \\
\hline & \multirow[t]{2}{*}{ MRI } & \multicolumn{3}{|c|}{ Model } & \multirow{2}{*}{$\begin{array}{c}\text { Ford } \\
\text { et al. } 45\end{array}$} & \multirow[t]{2}{*}{ MRI } & \multicolumn{3}{|c|}{ Model } & \multirow{2}{*}{$\begin{array}{c}\text { Ford } \\
\text { et al. } 45\end{array}$} \\
\hline & & $\begin{array}{c}\text { scenario } \\
\text { A }\end{array}$ & $\begin{array}{c}\text { scenario } \\
\text { B }\end{array}$ & $\begin{array}{c}\text { scenario } \\
\mathrm{C}\end{array}$ & & & $\begin{array}{c}\text { scenario } \\
\text { A }\end{array}$ & $\begin{array}{c}\text { scenario } \\
\text { B }\end{array}$ & $\begin{array}{c}\text { scenario } \\
\text { C }\end{array}$ & \\
\hline Right & $\begin{array}{c}6.18 \\
( \pm 0.90)\end{array}$ & $\begin{array}{c}6.05 \\
( \pm 0.89) \\
(\mathbf{2 . 1 2 \%})\end{array}$ & $\begin{array}{c}6.03 \\
( \pm 0.91) \\
(\mathbf{2 . 5 \%})\end{array}$ & $\begin{array}{c}6.07 \\
( \pm 0.90) \\
(\mathbf{1 . 8 \%})\end{array}$ & $\begin{array}{c}7.76 \\
( \pm 1.55)\end{array}$ & $\begin{array}{c}4.31 \\
( \pm 0.90)\end{array}$ & $\begin{array}{c}4.33 \\
( \pm 0.89) \\
(\mathbf{0 . 4 6 \%})\end{array}$ & $\begin{array}{c}4.25 \\
( \pm 0.91) \\
(\mathbf{1 . 4 \% )}\end{array}$ & $\begin{array}{c}4.32 \\
( \pm 0.90) \\
(\mathbf{0 . 2 3 \%})\end{array}$ & $\begin{array}{c}4.62 \\
( \pm 0.93)\end{array}$ \\
\hline Left & $\begin{array}{c}5.94 \\
( \pm 0.82)\end{array}$ & $\begin{array}{c}5.70 \\
( \pm 0.82) \\
(\mathbf{4 . 1 2 \%})\end{array}$ & $\begin{array}{l}5.61 \\
( \pm 0.80) \\
(\mathbf{5 . 7 1 \%})\end{array}$ & $\begin{array}{c}5.73 \\
( \pm 0.83) \\
(\mathbf{3 . 6 \%})\end{array}$ & $\begin{array}{c}7.35 \\
( \pm 1.72)\end{array}$ & $\begin{array}{c}4.13 \\
( \pm 0.82)\end{array}$ & $\begin{array}{c}4.18 \\
( \pm 0.82) \\
(\mathbf{1 . 2 \%})\end{array}$ & $\begin{array}{c}4.18 \\
( \pm 0.79) \\
(\mathbf{1 . 2 \%})\end{array}$ & $\begin{array}{c}4.18 \\
( \pm 0.83) \\
(\mathbf{1 . 2 \%})\end{array}$ & $\begin{array}{c}4.53 \\
( \pm 1.00)\end{array}$ \\
\hline
\end{tabular}




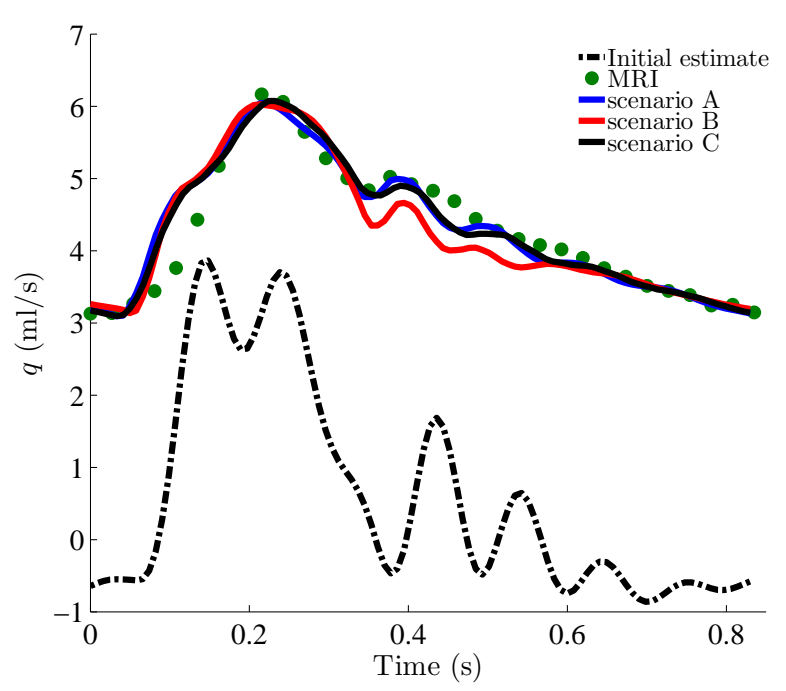

(a)

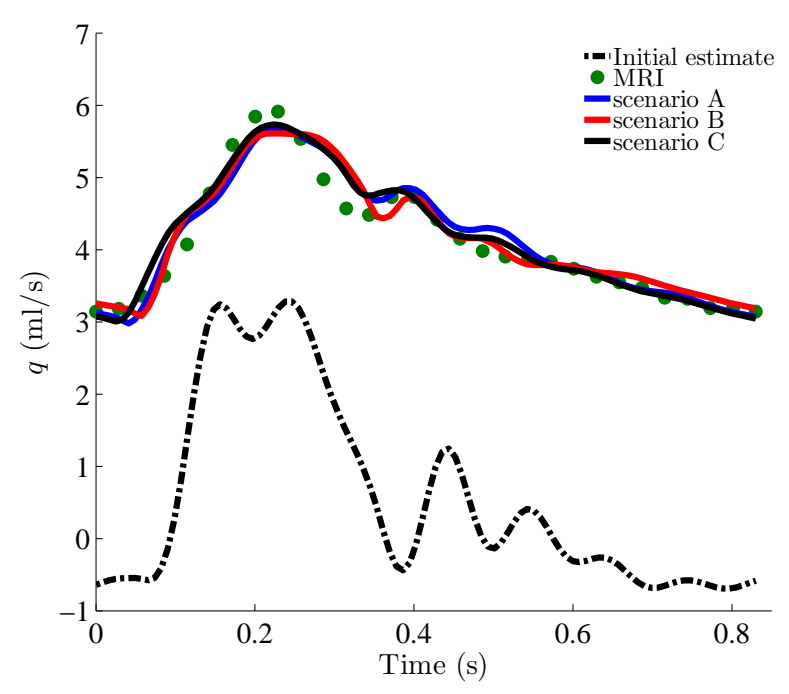

(b)

Figure 11: 11a Comparison of the model simulated blood flow rate waveform with the three scenarii: scenario A (in blue), scenario B (in red - as a posteriori validation), and scenario $\mathrm{C}$ (in black) to the target (MRI data in green) in the R-ICA. The dotted black line is the model simulation based on the initial estimate of parameters. The shaded areas represent the standard deviation around the ensemble mean values. (11b) shows the corresponding comparison of the blood flow in the L-ICA using scenario A (in blue as a posteriori validation), scenario B (in red), and scenario C (in black).

model. The predicted SBP and DBP in the arteries are all within the physiological values of a healthy patient.

The lack of clinically measured pressure in the brain prevents from validating the predicted cerebral pressure results. The clinical procedure to acquire pressure data in the cerebral arteries is mostly invasive in nature and requires inserting a pressure-sensing catheter from the femoral or radial arteries, e.g Hasan et al. $\frac{8}{}$ and Ferns et al. ${ }^{9}$. In clinical practice, the blood pressure in the brachial artery is usually measured at the upper arm. In this study, the accuracy of the model is assessed by comparing the predicted pressure results to the clinically measured SBP and DBP in the brachial artery. The following predicted values were obtained in the right brachial artery with the three scenarii $(\mathrm{mmHg})$ : $(125.3 \pm 1.7,70.9 \pm 1.7)$ with scenario A, $(126.1 \pm 1.7,70.6 \pm 1.7)$ with scenario B and $(124.7 \pm 1.6,71.3 \pm 1.6)$ with scenario C. In the left brachial artery, the final predicted values were $(126.6 \pm 1.7,70.9 \pm 1.7),(127.8 \pm 1.9,70.4 \pm 1.9)$ and $(125.7 \pm 1.6,71.0 \pm 1.6)$ with the scenarii $\mathrm{A}, \mathrm{B}$ and $\mathrm{C}$ respectively. It is recalled that the clinically measured pressure values in the right and the left brachial arteries are $(125,72)$ and $(115,72)$ respectively. The agreement between the predicted and measured pressure values is thus rather good. Note that the computed values obtained from the initial values of the 21 assimilated parameters are $(139,87)$ and $(138,87)$ in the right and left brachial arteries respectively. This shows the very positive impact of the data assimilation process in the derivation of a patient specific model. From this, and in absence of direct pressure measurements in the cerebral arteries, it is reasonable to believe that the predicted pressure in the upper region of the 
Predicted pressure range (diastolic to systolic)

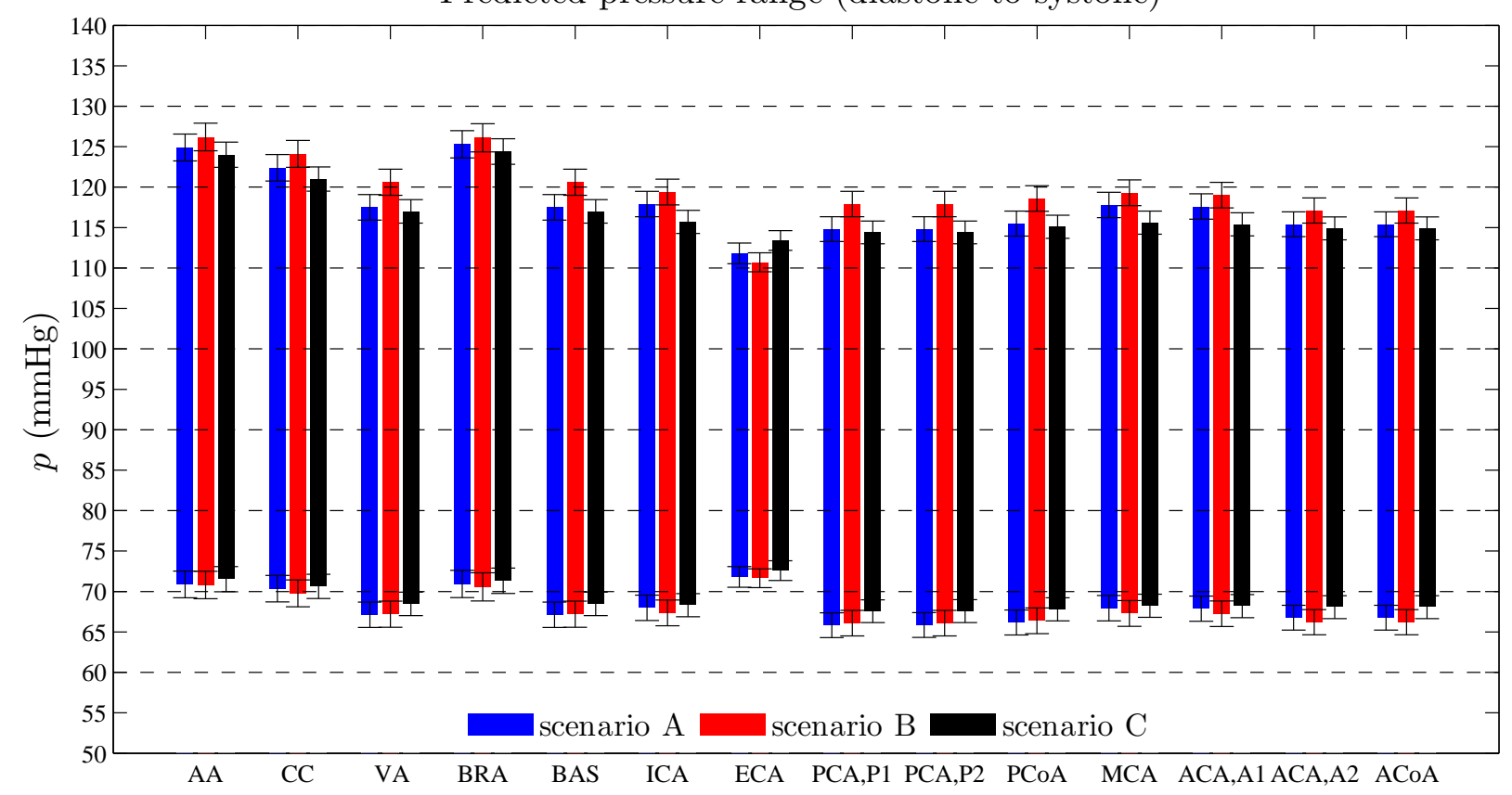

Figure 12: The systolic and diastolic blood pressures in the major arteries constituting the circle of Willis, vertebral arteries, and larger arteries as predicted using the three scenarii. The names of the arteries are written using acronyms which correspond to those in Table 2 . The data represent means \pm standard deviation.

network is also in fair agreement with its actual value. This is also supported by the fact that flow rate and pressure are physically linked through the model and that the computed flow rate matches well the observations.

One of the limitations could be the choice of the formula relating Young's modulus, thickness, and radius for the cerebral arteries. This work assumes the same relation for both large and cerebral arteries. The other factor might be the adaptation of missing data from the literature, such as the geometrical parameters of the arteries. The parameter estimation algorithm is only tested with a compartment model (reduced oder ODE) and not with a 1D blood flow model. This is because the observed computational cost of the PDE-based model for this configuration made it non feasible on standard computers.

Concerning the limitations and possible improvements, one could mention that the approach assumes zero pressure loss at bifurcations. Improvement in the results can also be brought with the use of different configurations of the 0D compartment numerical model. Westerhof et al. .20 demonstrated that the error made by lumping can be reduced (mostly for higher frequencies) by using the symmetrical network configuration ( $\pi$ element network) instead of using the inverse $\mathscr{L}$-type network. Future work includes studying the sensitivity of the predicted pressure to change in the model assumptions. One should however keep in mind that switching from $\mathscr{L}$ to $\pi$-type would increase the size of optimization problem by $3 / 2$ as there are 3 unknown parameters in each segment with $\pi$ instead of 2 for $\mathscr{L}^{19}$. Also, this study should be seen as a proof of concept as solely one clinical case has been considered. More extensive tests are definitely necessary before 
definite and final conclusions can be drawn.

\section{Conclusion}

A total of 21 parameters is estimated in a model of the circulation system of the circle of Willis, using clinical data (flow transients) from three points in the network (one as input of the model, another to define the cost function to be optimised, and the last to assess the quality of the fitting), and an advanced Kalman filtering approach (EnKF) to optimise the parameters.

To ease up the computational complexity and cost, a reduced order compartment model is coupled with the data assimilation algorithm, leading to an affordable CPU time of less than 3 hours for the most complex case on a standard computer. A relatively good-patient specific fitting is achieved even in the presence of partial geometrical parameters with values filled from the literature. The study demonstrates how to use the EnKF as an optimization tool to find parameters, and how to simplify the model and the number of variables to make the inverse hemodynamic problem tractable. The methodology seems to have a large potential in assessing patient-specific pressure waveforms at non-accessible (or difficult) locations in the cerebral arterial tree.

\section{Conflict of Interest}

The authors do not have any conflicts of interest to declare.

\section{Acknowledgment}

The authors greatly thank Dr. J. Siguenza for acting as a volunteer in the acquisition procedure.

Research done under the European Union Framework Programme Erasmus Mundus KITE (2013-2617/ 001-001 - EMA2)

\section{References}

1. Mathieu Sanchez, Dominique Ambard, Vincent Costalat, Simon Mendez, Franck Jourdan, and Franck Nicoud. Biomechanical assessment of the individual risk of rupture of cerebral aneurysms: a proof of concept. Annals of biomedical engineering, 41(1): 28-40, 2013.

2. Christopher L Taylor, Zhong Yuan, Warren R Selman, Robert A Ratcheson, and Alfred A Rimm. Cerebral arterial aneurysm formation and rupture in 20,767 elderly patients: hypertension and other risk factors. Journal of neurosurgery, 83(5):812-819, 1995.

3. D Mohan, V Munteanu, $\mathrm{T}$ Coman, and AV Ciurea. Genetic factors involves in intracranial aneurysms-actualities. Journal of medicine and life, 8(3):336, 2015. 
4. AWJ Hoksbergen, B Fülesdi, DA Legemate, and L Csiba. Collateral configuration of the circle of willis transcranial color-coded duplex ultrasonography and comparison with postmortem anatomy. Stroke, 31(6):1346-1351, 2000.

5. G Mulder, ACB Bogaerds, P Rongen, and FN van de Vosse. The influence of contrast agent injection on physiological flow in the circle of willis. Medical engineering $\&$ physics, 33(2):195-203, 2011.

6. J Alastruey, KH Parker, J Peiró, SM Byrd, and SJ Sherwin. Modelling the circle of willis to assess the effects of anatomical variations and occlusions on cerebral flows. Journal of biomechanics, 40(8):1794-1805, 2007.

7. Philippe Reymond, Fabrice Merenda, Fabienne Perren, Daniel Rüfenacht, and Nikos Stergiopulos. Validation of a one-dimensional model of the systemic arterial tree. American Journal of Physiology-Heart and Circulatory Physiology, 297(1):H208-H222, 2009.

8. David M Hasan, Bradley J Hindman, and Michael M Todd. Pressure changes within the sac of human cerebral aneurysms in response to artificially induced transient increases in systemic blood pressurenovelty and significance. Hypertension, 66(2):324-331, 2015.

9. SP Ferns, JJ Schneiders, M Siebes, R van Den Berg, ET van Bavel, and CB Majoie. Intracranial blood-flow velocity and pressure measurements using an intra-arterial dualsensor guidewire. American journal of neuroradiology, 31(2):324-326, 2010.

10. Gino I Montecinos, Lucas O Müller, and Eleuterio F Toro. Hyperbolic reformulation of a 1d viscoelastic blood flow model and ader finite volume schemes. Journal of Computational Physics, 266:101-123, 2014.

11. Etienne Boileau, Perumal Nithiarasu, Pablo J Blanco, Lucas O Müller, Fredrik Eikeland Fossan, Leif Rune Hellevik, Wouter P Donders, Wouter Huberts, Marie Willemet, and Jordi Alastruey. A benchmark study of numerical schemes for one-dimensional arterial blood flow modelling. International journal for numerical methods in biomedical engineering, 31(10), 2015.

12. Masashi Saito, Yuki Ikenaga, Mami Matsukawa, Yoshiaki Watanabe, Takaaki Asada, and Pierre-Yves Lagrée. One-dimensional model for propagation of a pressure wave in a model of the human arterial network: comparison of theoretical and experimental results. Journal of Biomechanical Engineering, 133(12):121005, 2011.

13. SA Urquiza, PJ Blanco, MJ Vénere, and RA Feijóo. Multidimensional modelling for the carotid artery blood flow. Computer Methods in Applied Mechanics and Engineering, 195(33):4002-4017, 2006.

14. Mauro Ursino and Massimo Giannessi. A model of cerebrovascular reactivity including the circle of willis and cortical anastomoses. Annals of biomedical engineering, 38(3): 955-974, 2010. 
15. Vuk Milišić and Alfio Quarteroni. Analysis of lumped parameter models for blood flow simulations and their relation with 1d models. ESAIM: Mathematical Modelling and Numerical Analysis, 38(4):613-632, 2004.

16. Sanjay Pant, Benoit Fabrèges, J-F Gerbeau, and IE Vignon-Clementel. A methodological paradigm for patient-specific multi-scale cfd simulations: from clinical measurements to parameter estimates for individual analysis. International journal for numerical methods in biomedical engineering, 30(12):1614-1648, 2014.

17. Laura M Ellwein, Hien T Tran, Cheryl Zapata, Vera Novak, and Mette S Olufsen. Sensitivity analysis and model assessment: mathematical models for arterial blood flow and blood pressure. Cardiovascular Engineering, 8(2):94-108, 2008.

18. Sanjay Pant, Chiara Corsini, Catriona Baker, Tain-Yen Hsia, Giancarlo Pennati, Irene E Vignon-Clementel, Modeling of Congenital Hearts Alliance (MOCHA) Investigators, et al. Data assimilation and modelling of patient-specific single-ventricle physiology with and without valve regurgitation. Journal of biomechanics, 49(11):2162-2173, 2016.

19. Alfio Quarteroni, Stefania Ragni, and Alessandro Veneziani. Coupling between lumped and distributed models for blood flow problems. Computing and Visualization in Science, $4(2): 111-124,2001$.

20. Nicolaas Westerhof, Frederik Bosman, Cornelis J De Vries, and Abraham Noordergraaf. Analog studies of the human systemic arterial tree. Journal of biomechanics, 2(2): 121IN1135IN3137IN5139-134136138143, 1969.

21. Pablo J Blanco, Raúl A Feijóo, et al. A 3d-1d-0d computational model for the entire cardiovascular system. Computational Mechanics, eds. E. Dvorking, M. Goldschmit, M. Storti, 29:5887-5911, 2010.

22. Fuyou Liang, Kazuaki Fukasaku, Hao Liu, and Shu Takagi. A computational model study of the influence of the anatomy of the circle of willis on cerebral hyperperfusion following carotid artery surgery. Biomedical engineering online, 10(1):84, 2011.

23. Erzhen Gao, William L Young, Eugene Ornstein, John Pile-Spellman, and Ma Qiyuan. A theoretical model of cerebral hemodynamics: application to the study of arteriovenous malformations. Journal of Cerebral Blood Flow \&G Metabolism, 17(8):905-918, 1997.

24. Mette Olufsen, Hien Tran, and Johnny Ottesen. Modeling cerebral blood flow control during posture change from sitting to standing. Cardiovascular engineering: an international journal, 4(1):47-58, 2004.

25. Scott R Pope, Laura M Ellwein, Cheryl L Zapata, Vera Novak, C Tim Kelley, and Mette S Olufsen. Estimation and identification of parameters in a lumped cerebrovascular model. Math Biosci Eng, 6(1):93-115, 2009.

26. Radomir Chabiniok, Philippe Moireau, P-F Lesault, Alain Rahmouni, J-F Deux, and Dominique Chapelle. Estimation of tissue contractility from cardiac cine-mri using a 
biomechanical heart model. Biomechanics and modeling in mechanobiology, 11(5):609630, 2012.

27. Sanjay Pant, Chiara Corsini, Catriona Baker, Tain-Yen Hsia, Giancarlo Pennati, and Irene E Vignon-Clementel. Inverse problems in reduced order models of cardiovascular haemodynamics: aspects of data assimilation and heart rate variability. Journal of The Royal Society Interface, 14(126):20160513, 2017.

28. Damiano Lombardi. Inverse problems in 1d hemodynamics on systemic networks: A sequential approach. International journal for numerical methods in biomedical engineering, 30(2):160-179, 2014.

29. Philippe Moireau, Cristobal Bertoglio, Nan Xiao, C Alberto Figueroa, CA Taylor, Dominique Chapelle, and J-F Gerbeau. Sequential identification of boundary support parameters in a fluid-structure vascular model using patient image data. Biomechanics and modeling in mechanobiology, 12(3):475-496, 2013.

30. Rajnesh Lal, Bijan Mohammadi, and Franck Nicoud. Data assimilation for identification of cardiovascular network characteristics. International Journal for Numerical Methods in Biomedical Engineering, 2016. doi: 10.1002/cnm.2824.

31. Kristen DeVault, Pierre A Gremaud, Vera Novak, Mette S Olufsen, Guillaume Vernieres, and Peng Zhao. Blood flow in the circle of willis: modeling and calibration. Multiscale Modeling \& Simulation, 7(2):888-909, 2008.

32. Cristóbal Bertoglio, Philippe Moireau, and Jean-Frederic Gerbeau. Sequential parameter estimation for fluid-structure problems: Application to hemodynamics. International Journal for Numerical Methods in Biomedical Engineering, 28(4):434-455, 2012.

33. A Caiazzo, F Caforio, G Montecinos, LO Muller, PJ Blanco, and EF Toro. Assessment of reduced-order unscented kalman filter for parameter identification in one-dimensional blood flow models using experimental data. International Journal for Numerical Methods in Biomedical Engineering, 2016.

34. Lucian Itu, Puneet Sharma, Tiziano Passerini, Ali Kamen, Constantin Suciu, and Dorin Comaniciu. A parameter estimation framework for patient-specific hemodynamic computations. Journal of Computational Physics, 281:316-333, 2015.

35. Laurent Dumas, Tamara El Bouti, and Didier Lucor. A robust and subject-specific hemodynamic model of the lower limb based on noninvasive arterial measurements. Journal of biomechanical engineering, 139(1):011002, 2017.

36. Peter L Houtekamer and Herschel L Mitchell. Data assimilation using an ensemble kalman filter technique. Monthly Weather Review, 126(3):796-811, 1998.

37. Youmin Tang, Jaison Ambandan, and Dake Chen. Nonlinear measurement function in the ensemble kalman filter. Advances in Atmospheric Sciences, 31(3):551-558, 2014. 
38. Mette S Olufsen. Structured tree outflow condition for blood flow in larger systemic arteries. American journal of physiology-Heart and circulatory physiology, 276(1):H257H268, 1999.

39. Mette S Olufsen, Ali Nadim, et al. On deriving lumped models for blood flow and pressure in the systemic arteries. Math Biosci Eng, 1(1):61-80, 2004.

40. N Stergiopulos, DF Young, and TR Rogge. Computer simulation of arterial flow with applications to arterial and aortic stenoses. Journal of biomechanics, 25(12):1477-1488, 1992.

41. Kevin Johnson, Puneet Sharma, and John Oshinski. Coronary artery flow measurement using navigator echo gated phase contrast magnetic resonance velocity mapping at 3.0 T. Journal of biomechanics, 41(3):595-602, 2008.

42. M Klarhöfer, B Csapo, Cs Balassy, JC Szeles, and E Moser. High-resolution blood flow velocity measurements in the human finger. Magnetic resonance in medicine, 45(4): 716-719, 2001.

43. Peter D Gatehouse, Marijn P Rolf, Karin Markenroth Bloch, Martin J Graves, Philip J Kilner, David N Firmin, and Mark BM Hofman. A multi-center inter-manufacturer study of the temporal stability of phase-contrast velocity mapping background offset errors. Journal of Cardiovascular Magnetic Resonance, 14(1):72, 2012.

44. Chao Tang, Duane D Blatter, and Dennis L Parker. Accuracy of phase-contrast flow measurements in the presence of partial-volume effects. Journal of Magnetic Resonance Imaging, 3(2):377-385, 1993.

45. Matthew D Ford, Noam Alperin, Sung Hoon Lee, David W Holdsworth, and David A Steinman. Characterization of volumetric flow rate waveforms in the normal internal carotid and vertebral arteries. Physiological Measurement, 26(4):477, 2005.

46. Aram V Chobanian, George L Bakris, Henry R Black, William C Cushman, Lee A Green, Joseph L Izzo, Daniel W Jones, Barry J Materson, Suzanne Oparil, Jackson T Wright, et al. Seventh report of the joint national committee on prevention, detection, evaluation, and treatment of high blood pressure. Hypertension, 42(6):1206-1252, 2003. 